\title{
Thermodynamics of Einstein-Proca AdS black holes
}

\author{
Hai-Shan Liu, ${ }^{a}$ H. Lï ${ }^{b}$ and C.N. Pope ${ }^{c, d}$ \\ ${ }^{a}$ Institute for Advanced Physics 8 Mathematics, Zhejiang University of Technology, \\ Hangzhou 310023, China \\ ${ }^{b}$ Department of Physics, Beijing Normal University, \\ Beijing 100875, China \\ ${ }^{c}$ George P. $\&$ Cynthia Woods Mitchell Institute for Fundamental Physics and Astronomy, \\ Texas A $6 M$ University, College Station, TX 77843, U.S.A. \\ ${ }^{d}$ DAMTP, Centre for Mathematical Sciences, Cambridge University, \\ Wilberforce Road, Cambridge CB3 OWA, U.K. \\ E-mail: hsliu.zju@gmail.com, mrhonglu@gmail.com, pope@physics.tamu.edu
}

ABSTRACT: We study static spherically-symmetric solutions of the Einstein-Proca equations in the presence of a negative cosmological constant. We show that the theory admits solutions describing both black holes and also solitons in an asymptotically AdS background. Interesting subtleties can arise in the computation of the mass of the solutions and also in the derivation of the first law of thermodynamics. We make use of holographic renormalisation in order to calculate the mass, even in cases where the solutions have a rather slow approach to the asymptotic AdS geometry. By using the procedure developed by Wald, we derive the first law of thermodynamics for the black hole and soliton solutions. This includes a non-trivial contribution associated with the Proca "charge". The solutions cannot be found analytically, and so we make use of numerical integration techniques to demonstrate their existence.

Keywords: Black Holes, AdS-CFT Correspondence, Classical Theories of Gravity

ARXIV EPRINT: 1402.5153 


\section{Contents}

1 Introduction $\quad 1$

2 Einstein-Proca AdS black holes 5

3 Holographic energy $\quad 8$

4 Thermodynamics from the Wald formalism 11

5 Solutions outside $0<\sigma \leq 1 \quad 14$

6 Numerical results $\quad 19$

$\begin{array}{lll}6.1 & \text { Solitonic solutions } & 20\end{array}$

6.2 Black hole solutions 23

$\begin{array}{llr}7 & \text { Conclusions } & 26\end{array}$

A Proca solutions in $\operatorname{AdS}_{n}$, and the Breitenlohner-Freedman bound $\quad 27$

\section{Introduction}

There has been a resurgence of interest recently in constructing black holes in a variety of theories that, in one way or another, are more general than those typically considered heretofore. One particular aspect that is now receiving considerable attention is the study of black holes in theories admitting ant-de Sitter (AdS) rather than Minkowski backgrounds, since asymptotically AdS solutions play a central role in the AdS/CFT correspondence [1-3].

Higher-derivative theories of gravity provide a fertile ground for constructing asymptotically AdS solutions, but they typically suffer from the drawback that the concomitant massive spin-2 modes have the wrong sign for their kinetic terms in a linearised analysis around an AdS background, and thus the theories are, in general, intrinsically plagued by ghosts. Nevertheless, as a framework for purely classical investigations, they can provide interesting starting points for the study of black-hole solutions and their dynamics. For example, in some recent work on the existence of black hole solutions in EinsteinWeyl gravity with a cosmological constant, it was found through numerical studies that asymptotically-AdS black holes can arise whenever the mass-squared $\mathbf{m}_{2}^{2}$ of the massive spin-2 mode is negative [6]. In general one may expect a negative mass-squared to exhibit another undesirable feature, namely the occurrence of tachyonic run-away instabilities that grow exponentially as a function of time. However, in anti-de Sitter backgrounds there is 
a "window" of negative mass-squared values $\mathbf{m}_{2 \mathrm{BF}}^{2} \leq \mathbf{m}_{2}^{2}<0$, where the negative masssquared $\mathbf{m}_{2 \mathrm{BF}}^{2}$ is the limiting value of the so-called Breitenlohner-Freedman bound $[4,5]$, above which the linearised modes in the AdS background still have oscillatory rather than real exponential time dependence, and thus the run-away behaviour is avoided.

In this paper, we shall study a different type of theory where again certain modes of non-tachyonic negative mass-squared play a central role, but now in the framework of a more conventional two-derivative theory that has no accompanying ghost problems. Specifically, we shall focus on the $n$-dimensional Einstein-Proca theory of a massive spin- 1 field coupled to Einstein gravity, in the presence also of a (negative) cosmological constant. ${ }^{1}$ This theory exhibits many features that are similar to those of a higher-derivative theory of gravity, with the massive Proca field now playing the rôle of the massive spin-2 mode. The solutions we study approach anti-de Sitter spacetime at large distance, with $R_{\mu \nu} \sim$ $-(n-1) \ell^{-2} g_{\mu \nu}$ as the radius tends to infinity. We find two distinct kinds of sphericallysymmetric static solutions, namely asymptotically AdS black holes, and smooth asymptotically AdS solitons. The metrics for both classes of solution take the form

$$
d s^{2}=-h(r) d t^{2}+\frac{d r^{2}}{f(r)}+r^{2} d \Omega_{n-2}^{2} .
$$

It is useful first to consider the situation where the mass is small and the Proca field is weak, so that its back-reaction on the spacetime geometry can be neglected. In this limit, the large-distance behaviour of the Proca potential $A=\psi(r) d t$ approaches that of a massive vector in AdS, which takes the form

$$
\psi(r) \rightarrow \frac{1}{r^{(n-3-\sigma) / 2}} \sum_{p=0}^{\infty} \frac{b_{p}}{r^{2 p}}+\frac{1}{r^{(n-3+\sigma) / 2}} \sum_{p=0}^{\infty} \frac{\tilde{b}_{p}}{r^{2 p}},
$$

where

$$
\sigma=\sqrt{4 \tilde{m}^{2} \ell^{2}+(n-3)^{2}},
$$

and $\tilde{m}$ is the mass of the Proca field. The Breitenlohner-Freedman window of non-tachyonic negative mass-squared values lies in the range $\mathbf{m}_{\mathrm{BF}}^{2} \leq \tilde{m}^{2}<0$, with

$$
\mathbf{m}_{\mathrm{BF}}^{2}=-\frac{1}{4} \ell^{-2}(n-3)^{2},
$$

thus ensuring that $\sigma$ remains real. The sums in (1.2) can actually be expressed in closed forms as hypergeometric functions (see appendix A).

It follows from the equations of motion that the back-reaction of the Proca field on the geometry will first appear in the metric functions $h$ and $f$ at order $1 / r^{n-3-\sigma}$, together with associated higher inverse powers of $r$. The back-reacted geometry will in turn modify

\footnotetext{
${ }^{1}$ Theories with a massive vector field coupled to gravity been considered extensively in discussions of holographic descriptions of non-relativistic boundary theories, arising from gravity duals where the spacetime approaches a Lifshitz or Schrödinger geometry. See, for example, [7-9]. In these cases the mass-squared of the massive vector field is taken to be positive.
} 
the Proca solution, with the onset beginning at order $1 / r^{(3 n-5-3 \sigma) / 2}$. Thus at large $r$ the Proca and metric functions will include terms of the general form

$$
\begin{aligned}
\psi & =\frac{q_{1}}{r^{(n-3-\sigma) / 2}}+\frac{q_{2}}{r^{(n-3+\sigma) / 2}}+\frac{a_{1} q_{1}^{3}}{r^{(3 n-5-3 \sigma) / 2}}+\cdots, \\
h & =r^{2} \ell^{-2}+1+\frac{m_{2}}{r^{n-3}}+\frac{a_{2} q_{1}^{2}}{r^{n-3-\sigma}} \cdots, \\
f & =r^{2} \ell^{-2}+1+\frac{n_{2}}{r^{n-3}}+\frac{a_{3} q_{1}^{2}}{r^{n-3-\sigma}} \cdots .
\end{aligned}
$$

The terms with coefficients $m_{2}$ and $n_{2}$ in $h$ and $f$ are associated with the mass of the black hole or soliton.

The ellipses denote all the remaining terms in the large- $r$ expansions. These will include the direct "descendants" of the $q_{1} / r^{(n-3-\sigma) / 2}$ and $q_{2} / r^{(n-3+\sigma) / 2}$ terms, at orders $1 / r^{(n-3-\sigma) / 2+2 p}$ and $1 / r^{(n-3+\sigma) / 2+2 p}$ for all integers $p \geq 1$, as in (1.2), and also higherorder back-reaction terms and descendants of these. Depending on the value of the index $\sigma$, defined by (1.3), some of these remaining terms may intermingle with orders already displayed in (1.5), or they may all be at higher orders than the displayed terms. The discussion of the asymptotic forms of the solutions can therefore become quite involved in general. It will be convenient for some of our calculations to focus the cases where $\sigma$ is sufficiently small that the displayed terms in (1.5) are in fact the leading order ones, and all the terms represented by the ellipses are of higher order than the displayed ones. This will certainly be the case, in all dimensions $n \geq 4$, if we choose

$$
\sigma \leq 1
$$

This allows us to investigate the asymptotic structure of the solutions systematically for a non-trivial range of Proca masses corresponding to $0 \leq \sigma \leq 1$, near to the BreitenlohnerFreedman bound.

In fact the special case when $\sigma=1$ is particularly nice, since then the characterisation of inverse powers of $r$ that arise in the asymptotic expansions of the Proca and metric functions is very simple. In this special case the leading-order term in the expansion of $\psi(r)$ is $1 / r^{(n-4) / 2}$, with each successive term having one extra power of $1 / r$. The terms in the metric functions occur always at integer powers of $1 / r$ :

$$
\begin{aligned}
& \psi(r)=\frac{1}{r^{(n-4) / 2}} \sum_{p=0}^{\infty} \frac{q_{p+1}}{r^{p}} \\
& h(r)=r^{2} \ell^{-2}+1+\frac{1}{r^{n-4}} \sum_{p=0}^{\infty} \frac{m_{p+1}}{r^{p}}, \quad f(r)=r^{2} \ell^{-2}+1+\frac{1}{r^{n-4}} \sum_{p=0}^{\infty} \frac{n_{p+1}}{r^{p}}
\end{aligned}
$$

Accordingly, we first study the asymptotic expansions in this special case with $\sigma=1$, where it is rather straightforward to see how one can systematically solve, order by order in powers of $1 / r$, for all the coefficients in terms of $q_{1}, q_{2}$ and $m_{2}$. We then look at the leading orders in the expansions for the general case $\sigma \leq 1$, sufficient for our subsequent purposes of computing the physical mass and deriving the first law of thermodynamics. 
We also study some isolated examples with $\sigma>1$, showing that even though the structure of the inverse powers of $r$ in the asymptotic expansions can then be rather involved, the solutions can still be found in these cases.

We then turn to the problem of calculating the physical mass of the solutions in terms of the free adjustable parameters of the asymptotic expansions, which can be taken to be $q_{1}$, $q_{2}$ and $m_{2}$. The calculation turns out to be somewhat non-trivial, because of the presence of the terms in the metric functions $h$ and $f$ at order $1 / r^{n-3-\sigma}$, which represents a slower fall-off than the usual $1 / r^{n-3}$ of a normal mass term in $n$ dimensions. Indeed, we find that a naive calculation using the prescription of Ashtekar, Magnon and Das (AMD) [10, 11], in which a certain electric component of the Weyl tensor in a conformally-related metric is integrated over the boundary $(n-2)$-sphere, leads to a divergent result. Presumably a more careful analysis, taking into account boundary contributions that can normally be neglected, may give rise to a finite and meaningful result. In the present paper we opt instead for calculating the mass using the method of the holographic stress tensor, and thereby obtain a well-defined finite result.

Having obtained an expression for the physical mass in terms of the parameters characterising the asymptotic form of the solution we then study the first law of thermodynamics, using the methods developed by Wald. We find that the Proca field makes a non-trivial contribution in the first law, which, for the static solutions we consider in this paper, will now take the form

$$
d M=T d S-\frac{\sigma \omega_{n-2}}{4 \pi} q_{1} d q_{2}
$$

where $\omega_{n-2}$ is the volume of the unit $(n-2)$-sphere. The way in which the Proca field contributes to the first law is analogous to a phenomenon that has been encountered recently when studying the thermodynamics of dyonic black holes in certain gauged supergravities, where parameters characterising the asymptotic behaviour of a scalar field enter in the first law, thus indicating the presence of a scalar "charge" or hair [12]. (See [13] for higher-dimensional generalisations.) In the present case, one can think of the asymptotic parameters $q_{2}$ and $q_{1}$ as being like a thermodynamic conjugate charge and potential pair characterising Proca "hair".

The equations of motion for the Einstein-Proca theory appear not to be exactly solvable even in the static spherically-symmetric situation that we study in this paper. We therefore turn to a numerical analysis in order to establish more explicitly the nature of the solutions to the theory. We do this by first developing small-distance series expansions for the metric and Proca functions, and then using these to set initial data for a numerical integration out to large distance. We find two different kinds of regular short-distance behaviour. In one type, we integrate out from a black-hole horizon located at some radius $r_{0}>0$ at which the Proca and metric functions all vanish. In the other type, we start from a smooth coordinate origin at $r=0$, where the Proca and metric functions all begin with non-vanishing constant values. In each of these types of solution, the numerical integration indicates that the fields stably approach the expected asymptotic forms we discussed above, thus lending confidence to the idea that such well-behaved black hole and soliton solutions do indeed exist. By matching the numerical solutions to the expansions (1.5) in the asymptotic region, we can 
relate the mass and Proca charge to black-hole area and surface gravity, and thereby obtain numerical confirmation of the first law (1.8).

The organisation of the paper is as follows. In section 2 we present the Lagrangian and equations of motion for the Einstein-Proca theory, and the consequent ordinary differential equations that are satisfied by the Proca potential and the metric functions in the static spherically-symmetric ansatz. We then examine the asymptotic forms of the solutions that arise when both of the parameters characterising the Proca field are turned on. In section 3, we show how the holographic renormalisation procedure may be used to calculate the mass of the solutions. In section 4, we use the formalism developed by Wald in order to derive the first law of thermodynamics for the black hole and soliton solutions. We first obtain results for values of the $\sigma$ parameter, defined in (1.3), lying in the range $0<\sigma \leq 1$. In section 5 we extend these calculations to values of $\sigma$ outside the $0<\sigma \leq 1$ range, showing how some new features can now arise. For example, we find that at certain values of $\sigma$, the confluence of generically-distinct inverse powers of $r$ can lead to the occurrence of logarithmic radial coordinate dependence in the solutions, which can then require new kinds of counterterm in order to cancel divergences. In section 6 , we carry out some numerical studies, in order to see how the asymptotic forms of the solutions we studied so far match onto the shortdistance forms that arise either near the horizon, in the case of black holes, or near the origin, in the case of solitons. The paper ends with conclusions in section 7. Some details of the exact static and spherically-symmetric solutions of the Proca equation in AdS are given in an appendix.

\section{Einstein-Proca AdS black holes}

We shall study black hole and soliton solutions in the $n$-dimensional Einstein-Proca theory of a massive vector field coupled to gravity, together with a cosmological constant. The Lagrangian, viewed as an $n$-form in $n$ dimensions, is given by

$$
\mathcal{L}=R * \mathbb{1}+(n-1)(n-2) \ell^{-2} * \mathbb{1}-2 * F \wedge F-2 \tilde{m}^{2} * A \wedge A
$$

This gives rise to the equations of motion

$$
\begin{aligned}
& E_{\mu \nu} \equiv R_{\mu \nu}-2\left(F_{\mu \nu}^{2}-\frac{1}{2(n-2)} F^{2} g_{\mu \nu}\right)-2 \tilde{m}^{2} A_{\mu} A_{\nu}+(n-1) \ell^{-2} g_{\mu \nu}=0, \\
& d * F=(-1)^{n} \tilde{m}^{2} * A .
\end{aligned}
$$

We shall consider spherically-symmetric solutions of the Einstein-Proca system, described by the ansatz

$$
d s^{2}=-h(r) d t^{2}+\frac{d r^{2}}{f(r)}+r^{2} d \Omega_{n-2}^{2}, \quad A=\psi(r) d t
$$


where $d \Omega_{n-2}^{2}$ is the metric of the unit $(n-2)$-sphere. The Ricci tensor of the metric in (2.4) is given by

$$
\begin{aligned}
& R_{t t}=h f\left(\frac{h^{\prime \prime}}{2 h}-\frac{h^{\prime 2}}{4 h^{2}}+\frac{h^{\prime} f^{\prime}}{4 h f}+\frac{(n-2) h^{\prime}}{2 r h}\right), \\
& R_{r r}=-\frac{h^{\prime \prime}}{2 h}+\frac{h^{\prime 2}}{4 h^{2}}-\frac{h^{\prime} f^{\prime}}{4 h f}-\frac{(n-2) f^{\prime}}{2 r f}, \\
& R_{i j}=\left((n-3)-\frac{r(h f)^{\prime}}{2 h}-(n-3) f\right) \tilde{g}_{i j},
\end{aligned}
$$

where $\tilde{g}_{i j}$ is the metric of the unit $(n-2)$-sphere. The equations of motion following from (2.2) and (2.3) can then be written as

$$
\begin{array}{rlrl}
\psi^{2} & =\frac{n-2}{4 \tilde{m}^{2} r}\left(f h^{\prime}-h f^{\prime}\right), & \left(E_{t}{ }^{t}-E_{r}^{r}=0\right), \\
-\frac{r(h f)^{\prime}}{2 h}-\frac{2 r^{2} f \psi^{\prime 2}}{(n-2) h}+(n-3)(1-f)+(n-1) \ell^{-2} r^{2} & =0, & & \left(E_{i}{ }^{j}=0\right), \\
\frac{\sqrt{h f}}{r^{n-2}}\left(r^{n-2} \sqrt{\frac{f}{h}} \psi^{\prime}\right)^{\prime} & =\tilde{m}^{2} \psi, & & \text { (Proca eom) } .
\end{array}
$$

The remaining equation, which may be taken to be $E_{t}{ }^{t}=0$, is in consequence automatically satisfied.

We shall be interested in studying two kinds of solutions of these equations, namely black holes and solitons. In the black hole solutions the functions $h(r)$ and $f(r)$ will both vanish on the horizon. The Proca equation implies that the potential $\psi$ will vanish on the horizon also. In the solitonic solutions the radial coordinate runs all the way down to $r=0$, which behaves as the origin of spherical polar coordinates. The functions $h, f$ and $\psi$ all approach constants at $r=0$.

It is not hard to see, by constructing power-series solutions of the equations near infinity, and also in the vicinity of a putative horizon at $r=r_{0}$, where one assumes that $h\left(r_{0}\right)=0$ and $f\left(r_{0}\right)=0$, that black holes could be expected to arise. Of course these series expansions do not settle the question of precisely how the interior and exterior solutions join together. This can be studied by means of a numerical integration of the equations, and we shall discuss this in greater detail in section 6 . For now, we just remark that the numerical analysis indeed confirms the existence of black-hole solutions.

Our immediate interest is in studying the asymptotic behaviour of the solutions, with a view to seeing how to calculate the mass of the black holes. This will also be relevant for studying the thermodynamics of the solutions. As we discussed in the introduction, a rather straightforward case arises if the mass $\tilde{m}$ of the Proc field is chosen so that the index $\sigma$, defined by (1.3), is equal to 1 . This means that the inverse powers of $r$ in the asymptotic expansions of the Proca and metric functions take the simple form given in (1.7). It is achieved by taking the mass of the Proca field to be given by

$$
\tilde{m}^{2}=-\frac{1}{4}(n-2)(n-4) \ell^{-2} .
$$


One can now systematically solve for the coefficients in the expansions (1.7) in terms of the free parameters $q_{1}, q_{2}$ and $m_{2}$. We find that the leading coefficients are given by

$$
n_{1}=\frac{1}{2} m_{1}=\frac{n-4}{n-2} q_{1}^{2}, \quad n_{2}=m_{2}-\frac{2(n-4)}{n-1} q_{1} q_{2} .
$$

At the next two orders, we find

$$
\begin{aligned}
q_{3} & =\frac{1}{8}(n-2)(n-4) \ell^{2} q_{1}, \quad n_{3}=q_{2}^{2}+\frac{1}{4}(n-2)(n-4) \ell^{2} q_{1}^{2}, \\
m_{3} & =\frac{2(n-2) q_{2}^{2}}{n}+\frac{(n-4)\left(n^{2}-6 n+10\right) \ell^{2} q_{1}^{2}}{2(n-2)}, \\
q_{4} & =\frac{1}{24}(n-2)(n-4) \ell^{2} q_{2}, \quad n_{4}=\frac{1}{3}(n-1)(n-4) \ell^{2} q_{1} q_{2}, \\
m_{4} & =\frac{(n-4)\left(2 n^{3}-8 n^{2}+7 n+11\right) \ell^{2} q_{1} q_{2}}{3\left(n^{2}-1\right)} .
\end{aligned}
$$

It is straightforward to continue the process of solving for the further coefficients to any desired order. In fact, for the purposes of computing the mass and deriving the first law of thermodynamics, it turns out to be unnecessary to go beyond the orders give in (2.8).

It should be noted that the specific choice (2.7) gives a rather natural higher-dimensional generalisation of the ordinary massless Einstein-Maxwell system in four dimensions. Setting $n=4$ we have $\tilde{m}=0$ and $\alpha=0$, and the Maxwell potential $A=\psi d t$ is simply given by

$$
\psi=q_{1}+\frac{q_{2}}{r} .
$$

Thus in this case $q_{2}$ is the ordinary electric charge of the Reissner-Nordström AdS black hole in four dimensions, and $q_{1}$ represents an arbitrary constant shift in the gauge potential.

In the more general case where the Proca mass is not fixed to the special value (2.7) that implies $\sigma=1$, but is instead allowed to lie anywhere in the range that corresponds to $\sigma \leq 1$ (see (1.3)), the leading orders in the asymptotic expansions take the form given in (1.5). Specifically, writing

$$
\begin{aligned}
\psi & =\frac{q_{1}}{r^{(n-3-\sigma) / 2}}+\frac{q_{2}}{r^{(n-3+\sigma) / 2}}+\cdots, \\
h & =r^{2} \ell^{-2}+1+\frac{m_{1}}{r^{n-3-\sigma}}+\frac{m_{2}}{r^{n-3}} \cdots, \\
f & =r^{2} \ell^{-2}+1+\frac{n_{1}}{r^{n-3-\sigma}}+\frac{n_{2}}{r^{n-3}}+\cdots,
\end{aligned}
$$

then substituting into the equations of motion we find

$$
\begin{aligned}
& n_{1}=\frac{n-3-\sigma}{n-2} q_{1}^{2}, \quad m_{1}=\frac{2(n-3-\sigma)}{n-1-\sigma} q_{1}^{2}, \\
& n_{2}=m_{2}-\frac{2(n-3-\sigma)(n-3+\sigma)}{(n-1)(n-2)} q_{1} q_{2} .
\end{aligned}
$$

One can continue solving for higher coefficients to any desired order in $1 / r$. Unlike the $\sigma=1$ case we discussed previously, this will in general be a somewhat less neatly ordered process, because of the intermingling of powers of $1 / r$ from different sources. However, it 
turns out for our present purposes, of calculating the mass and deriving the first law of thermodynamics, that the coefficients given in (2.12) are sufficient. Note that they reduce to those given in (2.8) in the case that $\sigma=1$.

The free parameter $m_{2}$ can be thought of as the "mass parameter" of the black hole or soliton; it is associated with the $r^{-(n-3)}$ fall-off in the metric coefficients $h(r)$ and $f(r)$. However, it is not itself directly proportional to the physical mass of the object. In fact, it can be seen from the expansions (2.11) that there is the potentially troubling feature that, unlike in a normal asymptotically-AdS black hole, here the metric functions have terms with a slower asymptotic fall off than the "mass terms", namely the $m_{1} / r^{n-3-\sigma}$ and $n_{1} / r^{n-3-\sigma}$ terms. Naively, these might be expected to give rise to an infinite result for the physical mass.

A simple and usually reliable way to calculate the mass of an asymptotically-AdS black hole is by means of the AMD procedure devised by Ashtekar, Magnon and Das [10, 11]. This involves integrating a certain "electric" component of the Weyl tensor over the sphere at infinity in an appropriate conformal rescaling of the metric. If we naively apply the AMD procedure to the solutions described above, we indeed obtain an infinite result for the physical mass. Rather than pursuing this further here, we shall instead use a different approach to calculating the physical mass, using the technique of the holographic stress tensor. This forms the subject of the next section.

\section{$3 \quad$ Holographic energy}

One way to calculate the mass of an asymptotically-AdS metric is by constructing the renormalised holographic stress tensor, via the AdS/CFT correspondence [14-17]. Thus one adds to the bulk Lagrangian given in (2.1) the standard Gibbons-Hawking surface term and the necessary holographic counterterms. At this stage it is more convenient to write the extra Lagrangian terms as scalar densities rather than as $n$-forms. Thus in the gravitational sector we have [16-18]

$$
\begin{aligned}
\mathcal{L}_{\text {bulk }}=\frac{1}{16 \pi G} \sqrt{-g}[ & \left.R-F^{\mu \nu} F_{\mu \nu}-2 \tilde{m}^{2} A^{\mu} A_{\mu}-(n-1) \ell^{-2}\right] \\
\mathcal{L}_{\text {surf }}=-\frac{1}{8 \pi G} \sqrt{-h} & K \\
\mathcal{L}_{\text {ct }}=\frac{1}{16 \pi G} \sqrt{-h}[ & -\frac{2(n-2)}{\ell}+\frac{\ell}{(n-3)} \mathcal{R}+b_{2} \ell^{3}\left(\mathcal{R}_{\mu \nu} \mathcal{R}^{\mu \nu}-\frac{(n-1)}{4(n-2)} \mathcal{R}^{2}\right) \\
& -b_{3} \ell^{5}\left(\frac{(3 n-1)}{4(n-2)} \mathcal{R}_{\mu \nu} \mathcal{R}^{\mu \nu}-\frac{\left(n^{2}-1\right)}{16(n-2)^{2}} \mathcal{R}^{3}-2 \mathcal{R}_{\mu \nu \rho \sigma} \mathcal{R}^{\mu \rho} \mathcal{R}^{\nu \sigma}\right. \\
& \left.\left.+\frac{(n-3)}{2(n-2)} \mathcal{R}^{\mu \nu} \nabla_{\mu} \nabla_{\nu} \mathcal{R}-\mathcal{R}^{\mu \nu} \square \mathcal{R}_{\mu \nu}+\frac{1}{2(n-2)} \mathcal{R} \square \mathcal{R}\right)+\cdots\right],
\end{aligned}
$$

where $K=h^{\mu \nu} K_{\mu \nu}$ is the trace of the second fundamental form $K_{\mu \nu}=-\nabla_{(\mu} n_{\nu)}, \mathcal{R}_{\mu \nu \rho \sigma}$ and its contractions denote curvatures in the boundary metric $h_{\mu \nu}=g_{\mu \nu}-n_{\mu} n_{\nu}$, and

$$
b_{2}=\frac{1}{(n-5)(n-3)^{2}}, \quad b_{3}=\frac{2}{(n-7)(n-5)(n-3)^{3}} .
$$


The ellipses in (3.3) denote terms of higher order in curvature or derivatives, which are only needed in dimensions $n>9$. The expressions in (3.3) should only be included when they yield divergent counterterms. This means that the terms with coefficient $c_{2}$ should be included only in dimensions $n>5$, and those with coefficient $c_{3}$ should be included only in dimensions $n>7$.

We should also include counterterms for the Proca field. There is, furthermore, an option also to add a boundary term for the Proca field, analogous to the Gibbons-Hawking term $\mathcal{L}_{\text {surf }}$ for the gravitational field. Thus we can take

$$
\mathcal{L}_{\text {surf }}^{A}=-\frac{\gamma}{8 \pi G} \sqrt{-h} n^{\mu} F_{\mu \nu} A^{\nu} .
$$

In the case of Dirichlet boundary conditions, where the value of the potential is fixed on the boundary, the coefficient $\gamma$ would be taken to be zero. For the counterterms, it turns out that for most of the solutions we shall be interested in we may simply take

$$
\mathcal{L}_{\text {ct }}^{A}=\frac{e_{1}}{16 \pi G \ell} \sqrt{-h} A^{\gamma} A_{\gamma} .
$$

We have left the constants $\gamma$ and $e_{1}$ arbitrary for now. It will turn out that one (dimensiondependent) linear combination of $\gamma$ and $e_{1}$ will be determined by the requirement of removing a divergence in the expression for the energy. The remaining combination then represents an ambiguity in the definition of the energy, corresponding to the freedom to perform a Legendre transformation to a different energy variable.

The variation of the surface and counterterms with respect to the boundary metric $h_{\mu \nu}$ gives the energy-momentum tensor $T_{\mu \nu}$ of the dual theory, with $T_{\alpha \beta}=(2 / \sqrt{-h}) \delta I / \delta h^{\alpha \beta}$. In our case, since we just wish to compute the energy, and since our metrics are sphericallysymmetric and static, many of the terms that come from the variations of the counterterms turn out to vanish. In particular, when we compute $T_{00}$ the only surviving contributions from the variations of the quadratic and cubic curvature terms will be those coming from the variation of $\sqrt{-h}$. This greatly simplifies the calculations. The upshot is that we may write

$$
\begin{aligned}
T_{\alpha \beta}=\frac{1}{8 \pi G}[ & K_{\alpha \beta}-K h_{\alpha \beta}-(n-2) \ell^{-1} h_{\alpha \beta}+\frac{\ell}{n-3}\left(\mathcal{R}_{\alpha \beta}-\frac{1}{2} \mathcal{R} h_{\alpha \beta}\right) \\
& -\frac{1}{2} b_{2} \ell^{3}\left(\mathcal{R}_{\mu \nu} \mathcal{R}^{\mu \nu}-\frac{(n-1)}{4(n-2)} \mathcal{R}^{2}\right) h_{\alpha \beta}+\frac{1}{2} b_{3} \ell^{5}\left(\frac{(3 n-1)}{4(n-2)} \mathcal{R} \mathcal{R}_{\mu \nu} \mathcal{R}^{\mu \nu}-\frac{\left(n^{2}-1\right)}{16(n-2)^{2}} \mathcal{R}^{3}\right. \\
& \left.-2 \mathcal{R}_{\mu \nu \rho \sigma} \mathcal{R}^{\mu \rho} \mathcal{R}^{\nu \sigma}+\frac{(n-3)}{2(n-2)} \mathcal{R}^{\mu \nu} \nabla_{\mu} \nabla_{\nu} \mathcal{R}-\mathcal{R}^{\mu \nu} \square \mathcal{R}_{\mu \nu}+\frac{1}{2(n-2)} \mathcal{R} \square \mathcal{R}\right) h_{\alpha \beta} \\
& \left.+\left(\gamma n^{\nu} F_{\mu \nu} A^{\nu}-\frac{1}{2} e_{1} \ell^{-1} A^{\gamma} A_{\gamma}\right) h_{\alpha \beta}-2 \gamma n^{\mu} F_{\mu(\alpha} A_{\beta)}+e_{1} \ell^{-1} A_{\alpha} A_{\beta}+\cdots\right]
\end{aligned}
$$

where the ellipses denote terms that will not contribute to $T_{00}$ for our solutions, and terms that are needed in dimensions $n>9$. The holographic mass is obtained by integrating $T_{00}$ over the volume of the $(n-2)$ sphere that forms the spatial boundary of the boundary metric. The boundary metric for our solutions, and the normal vector to the boundary, are given simply by

$$
h_{\mu \nu} d x^{\mu} d x^{\nu}=-h d t^{2}+r^{2} d \Omega_{n-2}^{2}, \quad n^{\mu} \partial_{\mu}=\sqrt{f} \frac{\partial}{\partial r} .
$$


We are now ready to insert the asymptotic expansions that we discussed earlier. We can use either (1.7), in the special case $\sigma=1$, or more generally (2.11) for all the cases with $\sigma \leq 1$. It turns out that the terms displayed in (2.11) are sufficient for the purpose, with the coefficients $m_{1}, n_{1}$ and $n_{2}$ given by (2.12).

Substituting into (3.7), we find that the counterterms subtract out all divergences, provided that we impose the relation

$$
e_{1}=(n-3-\sigma)(1-\gamma)
$$

on the two coefficients $\gamma$ and $e_{1}$ associated with the counterterms for the Proca field. We then find that the masses of the black holes in dimensions $5 \leq n \leq 9$ are given by ${ }^{2}$

$$
\begin{array}{ll}
n=5: & M=\frac{3 \pi}{8}\left[-m_{2}+\left(\frac{2}{3} \sigma \gamma+\frac{1}{6}(2-\sigma)(6+\sigma)\right) q_{1} q_{2}+\frac{1}{4} \ell^{2}\right] \\
n=6: & M=\frac{2 \pi}{3}\left[-m_{2}+\left(\frac{1}{2} \sigma \gamma+\frac{1}{10}(3-\sigma)(8+\sigma)\right) q_{1} q_{2}\right] \\
n=7: & M=\frac{5 \pi^{2}}{16}\left[-m_{2}+\left(\frac{2}{5} \sigma \gamma+\frac{1}{15}(4-\sigma)(10+\sigma)\right) q_{1} q_{2}-\frac{1}{8} \ell^{4}\right] \\
n=8: & M=\frac{2 \pi^{2}}{5}\left[-m_{2}+\left(\frac{1}{3} \sigma \gamma+\frac{1}{21}(5-\sigma)(12+\sigma)\right) q_{1} q_{2}\right] \\
n=9: & M=\frac{7 \pi^{3}}{48}\left[-m_{2}+\left(\frac{2}{7} \sigma \gamma+\frac{1}{28}(6-\sigma)(14+\sigma)\right) q_{1} q_{2}+\frac{5}{64} \ell^{6}\right] .
\end{array}
$$

Note that the term proportional to $\ell^{n-3}$ in each odd dimension is the Casimir energy. It would be natural to omit this if one wants to view the mass as simply that of a classical black hole.

Although the general expressions for the counterterms at the quartic or higher order in curvatures are not readily available, we can in fact easily calculate the holographic mass for the static spherically-symmetric solutions in any dimension. Any invariant constructed from $p$ powers of the curvature $\mathcal{R}_{\mu \nu \rho \sigma}$ of the boundary metric $h_{\mu \nu}$ will necessarily just be a pure dimensionless number times $r^{-2 p}$, and so the contributions $T_{00}^{\text {gct }}$ to $T_{00}$ coming from the gravitational counterterms to all orders can simply be written as

$$
T_{00}^{\mathrm{gct}}=\frac{1}{8 \pi G \ell} h_{00} \sum_{p=0}^{\infty} \frac{c_{p} \ell^{2 p}}{r^{2 p}} .
$$

The constants $c_{p}$ are then uniquely determined by the requirement of removing all divergences in the holographic expression for the mass. Together with the contributions from

\footnotetext{
${ }^{2}$ Our convention for the definition of mass in dimension $n$ is such that an ordinary AdS-Schwarzschild black hole, whose metric is given by (2.4) with $h=f=r^{2} \ell^{-2}+1-2 m r^{3-n}$, has mass $M=(n-2)$ $m \omega_{n-2} /(8 \pi)$, where

$$
\omega_{n-2}=\frac{2 \pi^{(n-1) / 2}}{\Gamma[(n-1) / 2]}
$$

is the volume of the unity $(n-2)$-sphere.
} 
the surface term and the counterterms for the Proca field, the complete expression for $T_{00}$ for our metrics is given by

$$
T_{00}=\frac{1}{8 \pi G}\left[-\frac{(n-2) h \sqrt{f}}{r}-\gamma \sqrt{f} \psi \psi^{\prime}+\frac{1}{2} e_{1} \ell^{-1} \psi^{2}+h \ell^{-1} \sum_{p=0}^{\infty} \frac{c_{p} \ell^{2 p}}{r^{2 p}}\right] .
$$

From this, we can calculate the mass of the Einstein-Proca black holes in arbitrary dimensions, finding in $n$ dimensions

$$
M=\frac{(n-2) \omega_{n-2}}{16 \pi}\left[-m_{2}+\left(b_{1}+b_{2} \gamma\right) q_{1} q_{2}\right]+E_{n}^{\text {Casimir }},
$$

where $e_{1}=(n-4)(1-\gamma)$ and

$$
b_{1}=\frac{2(n-3-\sigma)(2 n-4+\sigma)}{(n-1)(n-2)}, \quad b_{2}=\frac{2 \sigma}{n-2} .
$$

Note that if we write the energy in terms of the metric parameter $n_{2}$ rather than $m_{2}$, we obtain the simpler expression

$$
M=\frac{(n-2) \omega_{n-2}}{16 \pi}\left[-n_{2}+\frac{2(n-3-\sigma)+2 \sigma \gamma}{(n-2)} q_{1} q_{2}\right] .
$$

The Casimir energies are zero for even $n$, while for odd $n$ we find

$$
E_{n}^{\text {Casimir }}=\frac{(-1)^{(n-1) / 2} \pi^{(n-3) / 2} \ell^{n-3}(n-1)(n-2)(n-4) ! !}{2^{(n+3) / 2}([(n-1) / 2] !)^{2}} .
$$

\section{Thermodynamics from the Wald formalism}

Wald has developed a procedure for deriving the first law of thermodynamics by calculating the variation of a Hamiltonian derived from a conserved Noether current. The general procedure was developed in $[19,20]$. Its application in Einstein-Maxwell theory can be found in [21]. Starting from a Lagrangian $\mathcal{L}$, its variation under a general variation of the fields can be written as

$$
\delta \mathcal{L}=\text { e.o.m. }+\sqrt{-g} \nabla_{\mu} J^{\mu},
$$

where e.o.m. denotes terms proportional to the equations of motion for the fields. For the theory described by $(2.1), J^{\mu}$ is given by

$$
J^{\mu}=g^{\mu \rho} g^{\nu \sigma}\left(\nabla_{\sigma} \delta g_{\nu \rho}-\nabla_{\rho} \delta g_{\nu \sigma}\right)-4 F^{\mu \nu} \delta A_{\nu} .
$$

From this one can define a 1-form $J_{(1)}=J_{\mu} d x^{\mu}$ and its Hodge dual

$$
\begin{aligned}
& \Theta_{(n-1)}=(-1)^{n+1} * J_{(1)}=\Theta_{(n-1)}^{\mathrm{grav}}+\Theta_{(n-1)}^{A}, \\
& \Theta_{(n-1)}^{A}=-4(-1)^{n} * F \wedge \delta A .
\end{aligned}
$$

We now specialise to a variation that is induced by an infinitesimal diffeomorphism $\delta x^{\mu}=\xi^{\mu}$. One can show that

$$
J_{(n-1)} \equiv \Theta_{(n-1)}-i_{\xi} * L_{0}=\text { e.o.m. }-d * J_{(2)},
$$


where $i_{\xi}$ denotes a contraction of $\xi^{\mu}$ on the first index of the $n$-form $* L_{0}$, and

$$
J_{(2)}=-d \xi_{(1)}-4\left(i_{\xi} A\right) F,
$$

where $\xi_{(1)}=\xi_{\mu} d x^{\mu}$. One can thus define an $(n-2)$-form $Q_{(n-2)} \equiv * J_{(2)}$, such that $J_{(n-1)}=d Q_{(n-2)}$. Note that we use the subscript notation " $(p)$ " to denote a $p$-form. To make contact with the first law of black hole thermodynamics, we take $\xi^{\mu}$ to be the time-like Killing vector that is null on the horizon. Wald shows that the variation of the Hamiltonian with respect to the integration constants of a specific solution is given by

$$
\delta \mathcal{H}=\frac{1}{16 \pi} \delta \int_{c} J_{(n-1)}-\int_{c} d\left(i_{\xi} \Theta_{(n-1)}\right)=\frac{1}{16 \pi} \int_{\Sigma^{(n-2)}}\left(\delta Q_{(n-2)}-i_{\xi} \Theta_{(n-1)}\right),
$$

where $c$ denotes a Cauchy surface and $\Sigma^{(n-2)}$ is its boundary, which has two components, one at infinity and one on the horizon. In particular

$$
\delta Q_{(n-2)}^{A}-i_{\xi} \Theta_{(n-1)}^{A}=-4 i_{\xi} A * \delta F+4(-1)^{n} i_{\xi} * F \wedge \delta A .
$$

For the case of our ansatz (2.4), we find

$$
\begin{aligned}
Q_{(n-2)}^{\mathrm{grav}} & =h^{\prime} \sqrt{\frac{f}{h}} r^{n-2} \Omega_{(n-2)}, \\
Q_{(n-2)}^{\mathrm{A}} & =-4 \psi \psi^{\prime} \sqrt{\frac{f}{h}} r^{n-2} \Omega_{(n-2)}, \\
i_{\xi} \Theta_{(n-1)}^{\mathrm{grav}} & =r^{n-2}\left(\delta\left(h^{\prime} \sqrt{\frac{f}{h}}\right)+\frac{n-2}{r} \sqrt{\frac{h}{f}} \delta f\right) \Omega_{(n-2)}, \\
i_{\xi} \Theta_{(n-1)}^{\mathrm{A}} & =-4(\delta \psi) \psi^{\prime} \sqrt{\frac{f}{h}} r^{n-2} \Omega_{(n-2)} .
\end{aligned}
$$

In the asymptotic region at large $r$, this gives

$$
\delta Q-i_{\xi} \Theta=r^{n-2} \sqrt{\frac{h}{f}}\left(-\frac{n-2}{r} \delta f-\frac{4 f}{h} \psi \delta \psi^{\prime}-2 \psi \psi^{\prime}\left(\frac{\delta f}{h}-\frac{f \delta h}{h^{2}}\right)\right) \Omega_{(n-2)} .
$$

From the boundary on the horizon, one finds

$$
\frac{1}{16 \pi} \int_{r=r_{0}}\left(\delta Q-i_{\xi} \Theta\right)=T \delta S .
$$

(This result is a generalisation of that for Einstein-Maxwell theory obtained [21]. Analagous results were obtained in [13] for Einstein gravities coupled to a conformally massless scalar.)

Substituting the asymptotic expansions (2.11) into (4.9), we find from (4.6) that the contribution to $\delta \mathcal{H}$ from the boundary at infinity is given by sending $r$ to infinity in the expression

$$
\begin{aligned}
\delta \mathcal{H}_{\infty} \longrightarrow \frac{\omega_{n-2}}{16 \pi}\left\{r^{\sigma}\right. & {\left[-(n-2) \delta n_{1}+(n-3-\sigma) \delta q_{1}^{2}\right] } \\
& \left.-(n-2) \delta n_{1}+2(n-3-\sigma) q_{2} \delta q_{1}+2(n-3+\sigma) q_{1} \delta q_{2}\right\} .
\end{aligned}
$$


The ostensibly divergent $r^{\sigma}$ term in fact vanishes, by virtue of the relation between $n_{1}$ and $q_{1}$ given in (2.12).

If we now use our expression (3.16) for the holographic mass, the variation $\delta \mathcal{H}_{\infty}$ can be rewritten as

$$
\delta \mathcal{H}_{\infty}=\delta M+\frac{\sigma \omega_{n-2}}{4 \pi} q_{1} \delta q_{2}-\frac{\sigma \gamma \omega_{n-2}}{8 \pi} \delta\left(q_{1} q_{2}\right),
$$

and so, together with (4.10), we obtain the first law of thermodynamics in the form

$$
d M=T d S-\frac{\sigma \omega_{n-2}}{4 \pi} q_{1} d q_{2}+\frac{\sigma \gamma \omega_{n-2}}{8 \pi} d\left(q_{1} q_{2}\right) .
$$

It should be recalled that the parameter $\gamma$ can be chosen freely, with different choices corresponding to making Legendre transformations which redefine the mass, or energy, of the black hole by the addition of some constant multiple of $q_{1} q_{2}$ (see (3.14)). A simple choice is to take $\gamma=0$, in which case we have

$$
d M=T d S-\frac{\sigma \omega_{n-2}}{4 \pi} q_{1} d q_{2}
$$

As mentioned previously, taking $\gamma=0$ corresponds to the case where the potential at infinity is held fixed in the variational problem.

It is worth remarking that if we specialise to $\sigma=1$ in four dimensions, as we observed earlier, then $\tilde{m}^{2}=0$ and our Einstein-Proca model reduces to the ordinary EinsteinMaxwell system, then the $q_{1} d q_{2}$ term in (4.14) reduces simply to the standard $\Phi d Q$ contribution in the first law for charged black holes. Namely, $q_{2}$ is then electric charge $Q$, and the potential difference $\Phi=\Phi_{h}-\Phi_{\infty}$ is equal to $-q_{1}$, since in our general calculation the potential $\psi$ vanishes on the horizon and here, in four dimensions, the potential at infinity is equal to $q_{1}$ (see eqn (2.10)). More generally, choosing $\tilde{m}=0$ in any dimension $n$ gives $\sigma=n-3$. For this Einstein-Maxwell system we have $A=q_{1}+q_{2} r^{3-n}$, and again the first law (4.14) becomes the standard one for a charged black hole, derived in the gauge where the potential vanishes on the horizon.

Another choice for the constant $\gamma$ that might be considered natural is to choose it so that the $q_{1} d q_{2}$ and $q_{2} d q_{1}$ terms in the first law (4.13) occur in the ratio such that they vanish if one imposes a dimensionless relation between $q_{1}$ and $q_{2}$. Since these quantities have length dimensions $\left[q_{1}\right]=L^{(n-3-\sigma) / 2}$ and $\left[q_{2}\right]=L^{(n-3+\sigma) / 2}$, such a relation must take the form

$$
q_{1}^{n-3+\sigma}=c q^{n-3-\sigma},
$$

where $c$ is a dimensionless constant. Thus if we choose

$$
\gamma=\frac{n-3+\sigma}{n-3}
$$

then the first law (4.13) becomes

$$
d M=T d S-\frac{\sigma \omega_{n-2}}{8(n-3) \pi}\left[(n-3-\sigma) q_{1} d q_{2}-(n-3+\sigma) q_{2} d q_{1}\right]
$$

reducing simply to $d M=T d S$ if (4.15) is imposed. 
Finally in this section, we remark that the Wald type derivation of the first law that we discussed above can be applied also to the case of solitonic solutions to the EinsteinProca system. In these solutions there is no inner boundary, and instead $r$ runs outwards from $r=0$ which is simply like an origin in spherical polar coordinates. The behaviour of the metric and Proca functions at large $r$ takes the same general form as in (2.11). Thus when we apply the procedures described earlier in this section, we can derive a first law of thermodynamics that is just like the one for black holes, except that the $T d S$ term that came from the integral (4.10) over the boundary on the horizon. If we make the simple choice $\gamma$ defining the energy of the system, the first law (4.13) for the black hole case will simply be replaced by

$$
d M=-\frac{\sigma \omega_{n-2}}{4 \pi} q_{1} d q_{2}
$$

\section{Solutions outside $0<\sigma \leq 1$}

Until now, our discussion of the solutions to the Einstein-Proca system has concentrated on the cases where the Proca mass is such that the index $\sigma$, defined in (1.3), satisfies $\sigma \leq 1$. This was done in order to allow a relatively straightforward and uniform analysis of the asymptotic structure of the solutions. However, it should be emphasised that black hole and soliton solutions of the Einstein-Proca equations exist also if the index $\sigma$ lies in a wider range. Looking at the form of the expansions in (2.11), we see that the effects of the back-reaction of the Proca field on the metric components sets in at a leading order of $1 / r^{n-3-\sigma}$. Clearly, if this were to be of order $r^{2}$ or higher, then the back-reaction would be overwhelming the $r^{2} \ell^{-2}$ terms in $h$ and $f$ that establish the asymptotically-AdS nature of the solutions. Thus we can expect that in order to obtain asymptotically-AdS black holes or solitons, we should have

$$
\sigma<n-1
$$

which, from (1.3), implies that the Proca mass must satisfy

$$
\tilde{m}^{2}<m_{*}^{2} \equiv \frac{n-2}{\ell^{2}} .
$$

Thus the full Proca mass range where we may expect to find stable black hole and soliton solutions is

$$
-\frac{(n-3)^{2}}{\ell^{2}}<\tilde{m}^{2}<\frac{n-2}{\ell^{2}} .
$$

Below the Breitenlohner-Freedman bound which forms the lower limit, we expect the solutions to be unstable against time-dependent perturbations, on account of the tachyonic nature of the Proca field.

In the next section, where we carry out a numerical study of the various solutions, we find that indeed the upper bound in (5.3) represents the upper limit of where we appear to obtain well-behaved black hole and soliton solutions. At the lower end, the numerical integrations appear to be stable not only for the entire Breitenlohner-Freedman window of negative $\tilde{m}^{2}$ in (5.3), but also for arbitrarily negative $\tilde{m}^{2}$ below this, where the Proca field has become tachyonic. Presumably if we were to extend our numerical analysis to include 
the possibility of time-dependent behaviour we would find exponentially-growing timelike instabilities below the limit in (5.3), but these cannot be seen in the numerical integration of the static equations that we study here.

We may thus divide the range of possible values for $\tilde{m}^{2}$, the square of the Proca mass, as follows:

(1) $0<\tilde{m}^{2}<m_{*}^{2} ; \quad(n-3)<\sigma<(n-1)$

(2) $-\frac{1}{4}(n-2)(n-4) \ell^{-2} \leq \tilde{m}^{2}<0 ; 1 \leq \sigma<(n-3)$

(3) $m_{\mathrm{BF}}^{2}<\tilde{m}^{2}<-\frac{1}{4}(n-2)(n-4) \ell^{-2} ; \quad 0<\sigma<1$

(4) $\tilde{m}^{2}=\mathbf{m}_{\mathrm{BF}}^{2} ; \quad \sigma=0$

(5) $\tilde{m}^{2}<\mathbf{m}_{\mathrm{BF}}^{2} ; \quad \sigma$ imaginary

When the Proca mass lies in the range (1), the leading term in the fall-off in the metric functions $h$ and $f$ due to back reaction from the Proca field occurs at a positive power of $r$, lying between 0 and 2. In the range (2), the leading powers of $r$ in the metric functions due to back reaction are negative, and so the rate of approach to AdS is more conventional, but there can still be a rather complicated sequence of back-reaction terms at more dominant orders than the mass term $m_{2} / r^{n-3}$ in the metric functions. The range (3) corresponds to the cases we have already discussed in general, where the leadingorder terms in the asymptotic expansions take the form (1.5). A special case arises in (4), where the Proca mass-squared precisely equals the negative-most limit of the BreitenlohnerFreedman bound. Finally, in the range (5), the Proca mass-squared is more negative than the Breitenlohner-Freedman bound, and there is tachyonic behaviour.

It is instructive to examine some examples of the asymptotic behaviour of solutions where the Proca mass lies in the various ranges outside the cases in (3) that we have already studied. As a first example, we shall consider

Dimension $n=5$ with $\sigma=\frac{3}{2}$. Note that in this example, the mass-squared $\tilde{m}^{2}$ of the Proca field is still negative, with $\tilde{m}^{2}=-7 /\left(4 \ell^{2}\right)$, and it lies within the range (2) described above. We find that the asymptotic expansions for the Proca and metric functions take the form

$$
\begin{aligned}
\phi & =\frac{q_{1}}{r^{1 / 4}}+\frac{q_{2}}{r^{7 / 4}}+\frac{7 \ell^{2} q_{1}}{16 r^{9 / 4}}+\frac{7 \ell^{2} q_{1}^{3}}{120 r^{11 / 4}}+\frac{\ell^{2} q_{2}}{16 r^{15 / 4}}+\frac{7\left(16 \ell^{2} m_{2} q_{1}+22 \ell^{2} q_{1}^{2} q_{2}-9 \ell^{4} q_{1}\right.}{2560 r^{17 / 4}}+\cdots, \\
h & =r^{2} \ell^{-2}+1+\frac{2 q_{1}^{2}}{5 r^{1 / 2}}+\frac{m_{2}}{r^{2}}+\frac{49 \ell^{2} q_{1}^{2}}{60 r^{5 / 2}}+\frac{7 \ell^{2} q_{1}^{4}}{50 r^{3}}+\cdots, \\
f & =r^{2} \ell^{-2}+1+\frac{q_{1}^{2}}{6 r^{1 / 2}}+\frac{24 m_{2}-7 q_{1} q_{2}}{24 r^{2}}+\frac{35 \ell^{2} q_{1}^{2}}{48 r^{5 / 2}}+\frac{91 \ell^{2} q_{1}^{4}}{720 r^{3}}+\cdots .
\end{aligned}
$$

This example illustrates how when $\sigma>1$ we can get an intermingling of fall-off powers, with the $r^{-9 / 4}$ descendant of the leading $q_{1} r^{-1 / 4}$ term in the expansion for $\psi$ appearing prior to the first back-reaction term in (2.11), which is at order $r^{-11 / 4}$. Nevertheless, we find that the holographic mass and the Wald formula for the first law continue to give finite results which agree with the general expressions (3.14) and (4.13). 
As a second example we consider

Dimension $n=5$ with $\sigma=\frac{5}{2}$. In this case the Proca mass-squared is positive, with $\tilde{m}^{2}=+9 /\left(16 \ell^{2}\right)$. It is still less than the upper limit $\tilde{m}^{2}=m_{*}^{2}=3 / \ell^{2}$ in five dimensions that we described above, and so it lies within the range (1). The asymptotic forms of the Proca and metric functions are

$$
\begin{aligned}
& \phi=q_{1} r^{1 / 4}-\frac{\ell^{2} q_{1}^{3}}{8 r^{5 / 4}}+\frac{9 \ell^{2} q_{1}}{16 r^{7 / 4}}+\frac{q_{2}}{r^{9 / 4}}+\frac{21 \ell^{4} q_{1}^{5}}{128 r^{11 / 4}}+\cdots \\
& h=r^{2} \ell^{-2}-\frac{2}{3} q_{1}^{2} r^{1 / 2}+1-\frac{\ell^{2} q_{1}^{4}}{6 r}+\frac{\ell^{2} q_{1}^{2}}{4 r^{3 / 2}}+\frac{m_{2}}{r^{2}}+\frac{55 \ell^{4} q_{1}^{6}}{144 r^{5 / 2}}+\cdots, \\
& f=r^{2} \ell^{-2}-\frac{1}{6} q_{1}^{2} r^{1 / 2}+1-\frac{11 \ell^{2} q_{1}^{4}}{48 r}+\frac{9 \ell^{2} q_{1}^{2}}{16 r^{3 / 2}}+\frac{8 m_{2}+3 q_{1} q_{2}}{8 r^{2}}+\frac{229 \ell^{4} q_{1}^{6}}{576 r^{5 / 2}}+\cdots .
\end{aligned}
$$

In this case there is even more intermingling of the orders in the expansions, with the first back-reaction term in $\psi$, at order $r^{-5 / 4}$, preceding a descendant of the leading order $q_{1} r^{1 / 4}$ term and also preceding the second independent solution that begins with $q_{2} r^{-9 / 4}$. In the metric functions the first back-reaction term is at order $r^{1 / 2}$, which is prior even to the usual constant term of the pure AdS metric functions $\bar{h}=\bar{f}=r^{2} \ell^{-2}+1$.

A new feature that arises in this example is that we must now add further counterterms in the calculation of the holographic mass, in order to obtain a finite result. Specifically, we now need to include terms

$$
\mathcal{L}_{\mathrm{ct}}^{A, \text { extra }}=\frac{e_{2} \ell}{8 \pi G} \sqrt{-h}\left(A^{\mu} A_{\mu}\right)^{2}+\frac{e_{3} \ell}{8 \pi G} \sqrt{-h} \mathcal{R} A^{\mu} A_{\mu} .
$$

The divergences in the holographic mass are then removed if we take

$$
e_{1}=\frac{1}{2}(\gamma-1), \quad e_{2}=\frac{1}{18}(3-2 \gamma), \quad e_{3}=\frac{1}{3}(1-\gamma)
$$

The resulting expression for the holographic mass then agrees with the general formula (3.14), after specialising to $n=5$ and $\sigma=\frac{5}{2}$. Using the Wald procedure described in section 4 , we obtain the specialisation of (4.13) to $n=5$ and $\sigma=\frac{5}{2}$.

For another example, we consider

Dimension $n=6$ with $\sigma=2$. This lies within the range of category (2) above, and has $\tilde{m}^{2}=-5 /\left(4 \ell^{2}\right)$. Another new feature arises here, namely that we find also $\log r$ behaviour in the asymptotic expansions of the metric and Proca functions. To the first few orders at large $r$, we find

$$
\begin{aligned}
h & =\ell^{-2} r^{2}+1+\frac{2 q_{1}^{2}}{3 r}-\frac{5 \ell^{2} q_{1}^{2} \log r}{4 r^{3}}+\frac{m_{2}}{r^{3}} \cdots, \\
f & =\ell^{-2} r^{2}+1+\frac{q_{1}^{2}}{4 r}-\frac{15 \ell^{2} q_{1}^{2} \log r}{16 r^{3}}+\frac{n_{2}}{r^{3}}+\cdots, \\
\psi & =r^{-\frac{1}{2}}\left(q_{1}-\frac{5 \ell^{2} q_{1} \log r}{8 r^{2}}+\frac{q_{2}}{r^{2}}+\cdots\right) \\
m_{2} & =n_{2}+\frac{1}{2} q_{1} q_{2}-\frac{7}{48} \ell^{2} q_{1}^{2}
\end{aligned}
$$


The Wald formula still gives a convergent result, with

$$
\frac{16 \pi}{\omega_{4}} \delta \mathcal{H}_{\infty}=-4 \delta n_{2}+2 q_{2} \delta q_{1}+10 q_{1} \delta q_{2}+\frac{5}{2} \ell^{2} q_{1} \delta q_{1} .
$$

Now, however, using just the counterterms we have discussed so far we find that there is still an order $\log r$ divergence remaining in the holographic mass. It can be removed if we make a specific choice for the coefficient $\gamma$ in (3.5), namely $\gamma=1$. Thus in this case we are effectively obliged to view the surface term (3.5) as being instead a counterterm, which serves the purpose of removing the logarithmic divergence. We then find that all the divergences in the holographic mass are removed if we take

$$
e_{1}=0, \quad \gamma=1
$$

leading to

$$
M=\frac{2 \pi}{3}\left[-n_{2}+\frac{3}{2} q_{1} q_{2}+\frac{1}{16}\left(48 e_{3}+5\right) \ell^{2} q_{1}^{2}\right] .
$$

Note that although the $\sqrt{-h} \mathcal{R} A^{\mu} A_{\mu}$ counterterm in (5.6), with its coefficient $e_{3}$, is not required for the purpose of subtracting out any divergence, it is now making a contribution to the holographic mass, representing an ambiguity in its definition.

If we use (5.11) to eliminate $n_{2}$ from (5.9), this leads to the first law

$$
d M=T d S-\frac{2 \pi}{3}\left[3 q_{1} d q_{2}+q_{2} d q_{1}+6 e_{3} q_{1} d q_{1}\right] .
$$

A natural choice would be to take the free parameter $e_{3}$ to vanish.

For the next example, we consider case (4) above, where the Proca mass satisfies exactly the Breitenlohner-Freedman bound:

Dimension $n$ with $\sigma=0$. In $n$ dimensions, we therefore have $\tilde{m}^{2}=-(n-3)^{2} /\left(4 \ell^{2}\right)$. The large-distance expansions take the form

$$
\begin{aligned}
& h=\ell^{-2} r^{2}+1+\frac{m_{1}(\log r)^{2}+\tilde{m}_{1} \log r+m_{2}}{r^{n-3}}+\cdots, \\
& f=\ell^{-2} r^{2}+1+\frac{n_{1}(\log r)^{2}+\tilde{n}_{1} \log r+n_{2}}{r^{n-3}}+\cdots, \\
& \psi=\frac{q_{1} \log r+q_{2}}{r^{(n-3) / 2}}+\cdots, \quad m_{1}=2 n_{1}=\frac{2(n-3) q_{1}^{2}}{n-1}, \\
& \tilde{m}_{1}=\frac{4 q_{1}\left((n-1)(n-3) q_{2}-2 q_{1}\right)}{(n-1)^{2}}, \quad \tilde{n}_{1}=\frac{2 q_{1}\left((n-3) q_{2}-q_{1}\right)}{n-2}, \\
& m_{2}=n_{2}+\frac{(n-3)^{2}\left(2 q_{1}^{2}+2(n-1) q_{1} q_{2}+(n-1)^{2} q_{2}^{2}\right)}{(n-1)^{3}(n-2)} .
\end{aligned}
$$

We see that here also, there is logarithmic dependence on the $r$ coordinate in the asymptotic expansions. Nevertheless, the Wald formula turns out to be convergent, and we find

$$
\frac{16 \pi}{\omega_{n-2}} \delta \mathcal{H}_{\infty}=-(n-2) \delta n_{2}-4 q_{2} \delta q_{1}+2(n-3) q_{2} \delta q_{2} .
$$


Logarithmic divergences proportional to $\log r$ and $(\log r)^{2}$ arise in these $\sigma=0$ examples, and as in the earlier case of $\sigma=2$ in $n=6$ dimensions, it is necessary to make a specific choice for the coefficient $\gamma$ in the surface term (3.5) in order to remove them, namely by setting

$$
\gamma=\frac{4}{n-2} .
$$

Again it turns out that the original Proca counterterm (3.6) is not required in this case, and so we take $e_{1}=0$ here. The holographic mass is then given by

$$
M=\frac{(n-2) \omega_{n-2}}{16 \pi}\left[-n_{2}+\frac{1}{n-2}\left((n-3) q_{2}^{2}-2 q_{1} q_{2}\right) q_{2}\right],
$$

and hence from (5.14) we arrive at the first law

$$
d M=T d S-\frac{\omega_{n-2}}{8 \pi}\left(q_{1} d q_{2}-q_{2} d q_{1}\right) .
$$

The reason why $q_{1}$ and $q_{2}$ enter in a rather symmetrical way here may be related to the fact that in this $\sigma=0$ case the dimensions of the two quantities $q_{1}$ and $q_{2}$ are the same, as can be seen from the expansion for $\phi(r)$ in (5.13). Also, the different way in which the logarithmic singularities in the holographic mass are handled in these $\sigma=0$ examples could be related to the fact the that the original solution for $\phi$ itself, prior to taking back reactions into account, already has the logarithmic dependence associated with the coefficient $q_{1}$. Again, this arises because $q_{1}$ and $q_{2}$ have the same dimensions when $\sigma=0$. By contrast, in an example such as $n=6, \sigma=2$ discussed previously, the logarithmic dependences arose only via the back reactions, as a result of a confluence of powers of $r$ in these sub-leading terms.

Finally, we consider the cases (5) where the Proca mass-squared is more negative than the Breitenlohner-Freedman bound. We make take

Dimension $n$ with $\sigma=\mathrm{i} \tilde{\sigma}$. Here we have

$$
\tilde{\sigma}=\sqrt{-\frac{1}{4} \tilde{m}^{2} \ell^{2}-(n-3)^{2}}=-\mathrm{i} \sigma
$$

with $\tilde{m}^{2}<\mathbf{m}_{\mathrm{BF}}^{2}$ and so $\tilde{\sigma}$ is real. We find that the asymptotic expansions take the form

$$
\begin{aligned}
h & =\ell^{-2} r^{2}+1+\frac{m_{1} \cos (\tilde{\sigma} \log r)+\tilde{m}_{1} \sin (\tilde{\sigma} \log r)+m_{2}}{r^{n-3}}+\cdots, \\
f & =\ell^{-2} r^{2}+1+\frac{n_{1} \cos (\tilde{\sigma} \log r)+\tilde{n}_{1} \sin (\tilde{\sigma} \log r)+n_{2}}{r^{n-3}}+\cdots, \\
\psi & =\frac{q_{1} \cos \left(\frac{1}{2} \tilde{\sigma} \log r\right)+q_{2} \sin \left(\frac{1}{2} \tilde{\sigma} \log r\right)}{r^{(n-3) / 2}+\cdots,} \\
m_{1} & =\frac{\left(q_{1}^{2}-q_{2}^{2}\right) \tilde{\sigma}^{2}-4 q_{1} q_{2} \tilde{\sigma}+(n-1)(n-3)\left(q_{1}^{2}-q_{2}^{2}\right)}{\tilde{\sigma}^{2}+(n-1)^{2}}, \\
\tilde{m}_{1} & =\frac{2\left(q_{1}^{2}-q_{2}^{2}\right) \tilde{\sigma}+2 q_{1} q_{2}\left((n-1)(n-3)+\tilde{\sigma}^{2}\right)}{\tilde{\sigma}^{2}+(n-1)^{2}}, \\
n_{1} & =\frac{(n-3)\left(q_{1}^{2}-q_{2}^{2}\right)-2 q_{1} q_{2} \tilde{\sigma}}{2(n-2)},
\end{aligned}
$$




$$
\begin{aligned}
\tilde{n}_{1} & =\frac{\left(q_{1}^{2}-q_{2}^{2}\right) \tilde{\sigma}+2(n-3) q_{1} q_{2}}{2(n-2)}, \\
m_{2} & =n_{2}+\frac{\left(q_{1}^{2}+q_{2}^{2}\right)\left((n-3)^{2}+\tilde{\sigma}^{2}\right)}{2(n-1)(n-2)} .
\end{aligned}
$$

The resulting Wald formula is given by

$$
\frac{16 \pi}{\omega_{n-2}} \delta \mathcal{H}_{\infty}=-(n-2) \delta n_{2}+\tilde{\sigma}\left(q_{2} \delta q_{1}-q_{1} \delta q_{2}\right)+\frac{1}{2}(n-3) \delta\left(q_{1}^{2}+q_{2}^{2}\right) .
$$

Again, as in the previous example, the original Proca counterterm (3.6) is not required to regularize the holographic mass, and so we may take $e_{1}=0$. Terms proportional to $\sin (\tilde{\sigma} \log r)$ and $\cos (\tilde{\sigma} \log r)$ can be removed by taking the coefficient $\gamma$ of the Proca surface term (3.5) to be again given by (5.15). This yields the finite result

$$
M=\frac{(n-2) \omega_{n-2}}{16 \pi}\left[-n_{2}+\frac{(n-3)}{2(n-2)}\left(q_{1}^{2}+q_{2}^{2}\right)\right]
$$

for the holographic mass, and hence, from (5.20), we arrive at the first law

$$
d M=T d S-\frac{\tilde{\sigma} \omega_{n-2}}{16 \pi}\left(q_{2} d q_{1}-q_{1} d q_{2}\right) .
$$

\section{$6 \quad$ Numerical results}

It does not appear to be possible to solve the Einstein-Proca equations of motion for static spherically-symmetric geometries analytically, and so we now resort to numerical integration in order to gain more insight into the solutions. Two distinct kinds of regular solutions can arise; firstly black holes, and secondly what we shall refer to as "solitons".

The black hole solutions can be found numerically by first assuming that there exists an horizon at some radius $r=r_{0}$, at which the metric functions $h(r)$ and $f(r)$ vanish, then performing Taylor expansions of the metric and Proca field functions $h(r), f(r)$ and $\psi(r)$ around the point $r_{0}$, and then using these expansions to set initial conditions just outside the horizon for a numerical integration out to infinity. The criterion for obtaining a "regular" black hole solution is that the functions should smoothly and stably approach the asymptotic forms (1.7) that we assumed in our discussion in section 2. Since, as we have seen, the general asymptotic solutions, with all three independent parameters $q_{1}, q_{2}$ and $m_{2}$ nonvanishing, are well-behaved at infinity (provided the Proc mass $\tilde{m}$ satisfies $\tilde{m}^{2}<m_{*}^{2}$, where $m_{*}$ is defined in eqn (5.2), there is no reason why a solution that is well-behaved on the horizon will not integrate out smoothly to a well-behaved solution at infinity, and indeed, that is what we find in the numerical analysis. ${ }^{3}$

\footnotetext{
${ }^{3}$ Note that the situation would be very different in the absence of a cosmological constant. The asymptotic form of the Proca solutions is then given by (A.6), and so one of the two solutions diverges exponentially at infinity, assuming $\tilde{m}^{2}>0$. The analogous evolution from a well-behaved starting-point on the horizon would then inevitably pick up the diverging solution at infinity, leading to a singular behaviour. The exponential divergence could be avoided if $\tilde{m}^{2}$ were negative, but in a Minkowski background this would always be tachyonic, and so there would be instabilities because of exponential run-away behaviour as a function of time.
} 
The solitonic solutions have a very different kind of interior behaviour, in which the functions $h(r), f(r)$ and $\psi(r)$ all approach constant values at the origin at $r=0$. To study these numerically we start by obtaining small- $r$ expansions for the functions, using these to set initial conditions just outside the origin, and then integrating out to large $r$. The asymptotic forms of the metric and Proca functions will again be of the general form given in (1.7) in the case of smooth solitonic solutions. Again, since the generic asymptotic solutions with Proca mass satisfying $\tilde{m}^{2}<m_{*}^{2}$ are well-behaved, the smooth solutions near the origin will necessarily evolve to solutions that are well-behaved at infinity. Since the solitonic solutions are somewhat simpler than the black holes, we shall begin first by investigating the solitons. For the rest of this section, we shall, without loss of generality, set the AdS scale size by taking

$$
\ell=1
$$

\subsection{Solitonic solutions}

The soliton solutions we are seeking have no boundary at small $r$; rather, $r=0$ will be like the origin of spherical polar coordinates. We begin by making Taylor expansions for the metric and Proca functions, taking the form

$$
\begin{aligned}
& h=\alpha\left(1+b_{2} r^{2}+b_{4} r^{4}+\cdots\right), \quad f=1+c_{2} r^{2}+c_{4} r^{4}+\cdots, \\
& \psi=\sqrt{\alpha}\left(a_{0}+a_{2} r^{2}+a_{4} r^{4}+\cdots\right) .
\end{aligned}
$$

Substituting into the equations of motion (2.6), one can systematically solve for the coefficients $\left(a_{2}, a_{4}, \ldots\right),\left(b_{2}, b_{4}, \ldots\right)$ and $\left(c_{2}, c_{4}, \ldots\right)$ in terms of the coefficient $a_{0}$. Thus we find

$$
\begin{aligned}
& a_{2}=-\frac{(n-2)(n-4) a_{0}}{8(n-1)}, \quad b_{2}=\frac{2(n-1) \ell^{-2}-(n-2)(n-4) a_{0}^{2}}{2(n-1)}, \\
& c_{2}=\frac{2(n-1) \ell^{-2}+a_{0}^{2}(n-4)}{2(n-1)},
\end{aligned}
$$

with progressively more complicated expressions for the higher coefficients that we shall not present explicitly here. The coefficient $\alpha$ represents the freedom to rescale the time coordinate. In an actual numerical calculation, when we integrate out to large $r$, we may, without loss of generality, start out by choosing $\alpha=1$, and then, by taking the limit

$$
\lim _{r \rightarrow \infty} \frac{h}{r^{2}}=\beta \text {, }
$$

determine the appropriate scaling factor that allows us the to redefine our $\alpha$ by setting $\alpha=1 / \beta$. Thus we see that the soliton solution has only the one free parameter, $a_{0}$.

To illustrate the numerical integration process, let us consider the example of the soliton in dimension $n=5$. We determined the coefficients in the expansions (6.2) up to the $r^{8}$ order, and used these to set initial conditions for integrating the equations (2.6) out to large $r$. We found that indeed the solitons, parameterised by the constant $a_{0}$, have a well-behaved and stable asymptotic behaviour, in which the functions $h, f$ and $\psi$ approach the forms given in (1.7) with $n=5$. In particular, we have

$$
h \rightarrow r^{2}+1+\frac{m_{1}}{r}+\frac{m_{2}}{r^{2}}+\cdots, \quad \psi \rightarrow \frac{q_{1}}{r^{1 / 2}}+\frac{q_{2}}{r^{3 / 2}}+\cdots .
$$

(Recall that we are setting the AdS scale $\ell=1$ in this section.) 

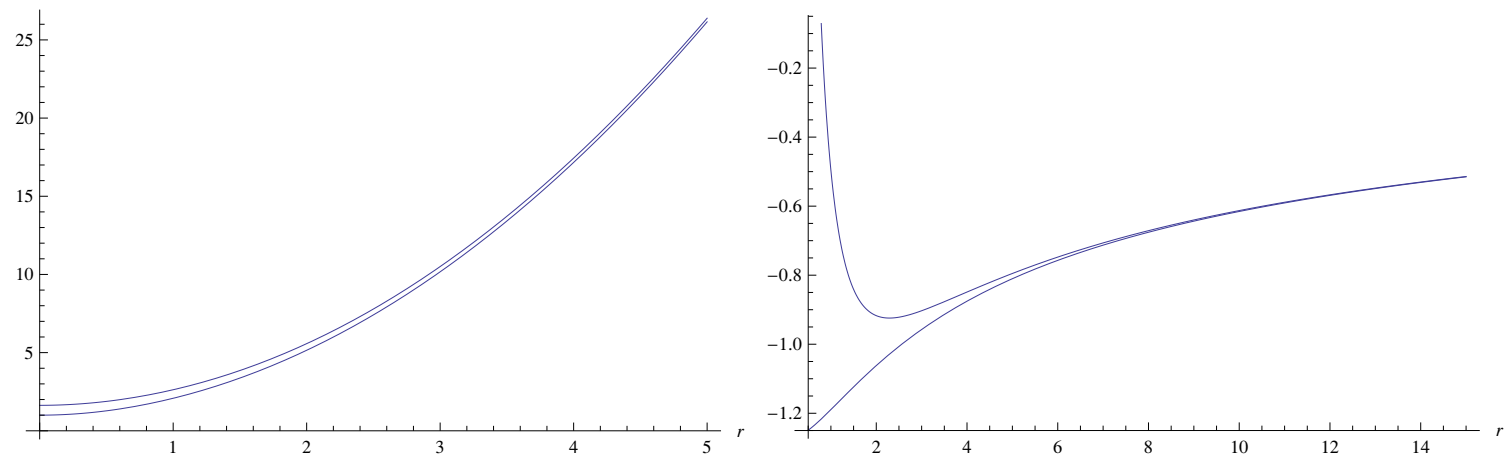

Figure 1. Smooth soliton in $n=5$ dimensions, with $a_{0}=-1$ and $\alpha=1.6285$. The left-hand plot shows the metric functions $h(r)$ and $f(r)$. The upper line is $h(r)$, starting from $h(0)=\alpha$, and the lower line is $f(r)$, starting from $f(0)=1$. To leading order, they coalesce at large $r$. The right-hand plot shows the potential function $\psi$ (lower line), and compares it with a best fit of the function $\tilde{\psi}=q_{1} / r^{\frac{1}{2}}+q_{2} / r^{\frac{3}{2}}$ (upper line) that represents the leading-order terms in the large- $r$ expansion in (6.2), achieved by taking $q_{1}=-2.0980$ and $q_{2}=1.6018$. The numerically-obtained potential function $\psi$ converges at small $r$ to $\psi(0)=\sqrt{\alpha}$.

In principle, for a given solitonic solution determined by the choice of the free parameter $a_{0}$, we can match the numerically-determined asymptotic form of the solution to the expansions (6.5), and hence read off the values of the coefficients $q_{1}, q_{2}, m_{1}$ and $m_{2}$. It is quite delicate to do this, especially to pick up the coefficient $m_{2}$ which occurs at four inverse powers of $r$ down from the leading-order behaviour of $h(r)$. A useful guide to the accuracy of the integration routine is to match the numerical results for $h(r)$ at large $r$ to an assumed form

$$
h=\gamma_{0} r^{2}+\gamma_{1}+\frac{m_{1}}{r}+\frac{m_{2}}{r^{2}} .
$$

Ideally, one should find $\gamma_{0}=1$ and $\gamma_{1}=1$. There will in fact, of course, be errors. We first rescale the numerically-determined $h(r)$ and $\psi(r)$ by the factors $1 / \gamma_{0}$ and $1 / \sqrt{\gamma_{0}}$ respectively. A test of a reliable solution is then that to high accuracy we should find $($ see $(2.8))$

$$
\gamma_{1}-1 \sim 0, \quad m_{1}-\frac{2}{3} q_{1}^{2} \sim 0
$$

We now present an explicit example of a smooth soliton solution, for which we shall make the choice with $a_{0}=-1$ in (6.2). (We choose a negative value of $a_{0}$ so that $q_{2}$ is positive, and in fact $q_{2}>0>q_{1}$.) For this choice of $a_{0}$, we find that the free scaling parameter $\alpha$ should be chosen to be $\alpha=1.6285$ in order to ensure that $\beta=1.0000$. The behaviour of the metric functions $(h, f)$ and the potential function $\psi$ is then displayed in figure 1. In the left-hand plot, can be seen that the metric functions are indeed running smoothly from constant values at the origin, and at large $r$ they approach their expected AdS forms. By a careful matching of the asymptotic forms of the function $h(r)$ to the expansion (6.2), we can read off a value for the "mass" parameter $m_{2}$, finding $m_{2}=$ -6.2630 . 

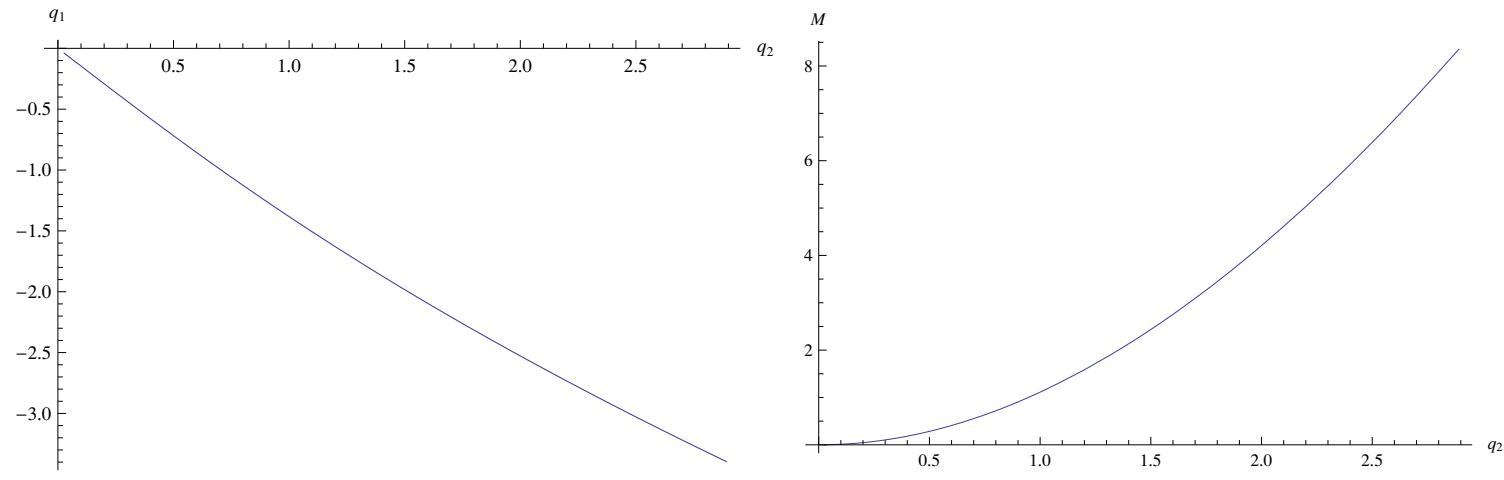

Figure 2. The left-hand plot shows $q_{1}$ as a function of $q_{2}$, whilst the right-hand plot shows the mass $M$ as a function of $q_{2}$, for $0<q_{2}<3$. Correspondingly, $a_{0}$ runs from $a_{0}=0+$ to $a_{0}=-1.3$.

Repeating this calculation for a range of values for $a_{0}$, we obtain reasonably trustworthy numerical expressions for

$$
m_{2}=m_{2}\left(a_{0}\right), \quad q_{1}=q_{1}\left(a_{0}\right), \quad q_{2}=q_{2}\left(a_{0}\right) .
$$

We can then choose to use $q_{2}$ to parameterise the solutions, rather than $a_{0}$, so that we can write

$$
m_{2}=m_{2}\left(q_{2}\right), \quad q_{1}=q_{1}\left(q_{2}\right) .
$$

We are now in a position to attempt a numerical verification of the first law of thermodynamics for the solitonic solutions. Choosing the parameter $\gamma$ in section 4 to be zero for simplicity, the energy of the five-dimensional soliton is given, from (3.11), by

$$
M=\frac{3 \pi}{8}\left(-m_{2}+\frac{7}{6} q_{1} q_{2}\right) .
$$

Viewing $M$ and $q_{1}$ as functions of $q_{2}$, we can now check how accurately the first law (4.18), which in $n=5$ dimensions reads

$$
d M=-\frac{1}{2} \pi q_{1} d q_{2},
$$

is satisfied. In figure 2, we display plots of $q_{1}\left(q_{2}\right)$ and $M\left(q_{2}\right)$ for a range of $q_{2}$ values with $0<q_{2}<3$.

After fitting the data for these curves, we obtain the approximate relations

$$
q_{1}=-1.486 q_{2}+0.1094 q_{2}^{2}, \quad M=\frac{1}{2} \pi\left(0.7449 q_{2}^{2}-0.03764 q_{2}^{3}\right) .
$$

Thus we find

$$
-\frac{2}{\pi} \frac{\partial M}{\partial q_{2}}=-1.490 q_{2}+0.1129 q_{2}^{2}
$$

which should, according to (6.11), be equal to $q_{1}$. It is indeed in reasonable agreement with the approximate form for $q_{1}$ given in (6.12).

It is worth pointing out that as we increase the negative value of $a_{0}$, the mass becomes divergent, with the solution becoming singular around $a_{0}=-1.8$. 


\subsection{Black hole solutions}

The static black hole solutions that we are seeking are characterised by the fact that the Killing vector $\partial / \partial t$ will become null on the horizon at $r=r_{0}$. Thus the metric function $h(r)$ in (2.4) will have a zero at $r=r_{0}$. It follows from the equations of motion (2.6) that the functions $f(r)$ and $\psi(r)$ will vanish at $r=r_{0}$ also. We are therefore led to consider near-horizon series expansions of the form

$$
\begin{aligned}
h & =b_{1}\left[\left(r-r_{0}\right)+b_{2}\left(r-r_{0}\right)^{2}+\cdots\right], \\
f & =c_{1}\left(r-r_{0}\right)+c_{2}\left(r-r_{0}\right)^{2}+\cdots, \\
\psi & =\sqrt{b_{1}}\left[a_{1}\left(r-r_{0}\right)+a_{2}\left(r-r_{0}\right)^{2}+\cdots\right] .
\end{aligned}
$$

The constant $b_{1}$ parameterises the freedom to rescale the time coordinate by a constant factor. It can be used in order to rescale the solution, after numerical integration out to large distances, so that the time coordinate is canonically normalised.

Substituting the expansions (6.14) into the equations of motion (2.6), we can systematically solve for the coefficients $\left(a_{2}, a_{3}, \ldots\right),\left(b_{2}, b_{3}, \ldots\right)$ and $\left(c_{1}, c_{2}, \ldots\right)$ in terms of $r_{0}$ and $a_{1}$. In dimension $n$ the solution for $c_{1}$ is

$$
c_{1}=\frac{(n-2)(n-3)+(n-1) r_{0}^{2}}{\left(2 r_{0} a_{1}^{2}+n-2\right) r_{0}} .
$$

(Recall, again, that we have set the AdS scale $\ell=1$ in this section.) The expressions for the higher coefficients are all quite complicated in general dimensions. Here, as an example, we just present $\left(a_{2}, b_{2}, c_{2}\right)$ in the special case of $n=5$ :

$$
\begin{aligned}
& a_{2}=-\frac{a_{1}\left(4 r_{0}^{4} a_{1}^{4}+12 r_{0}^{3} a_{1}^{2}+153 r_{0}^{2}+72\right)}{48 r_{0}\left(2 r_{0}^{2}+1\right)}, \\
& b_{2}=-\frac{2 r_{0}^{4} a_{1}^{4}-93 r_{0}^{3} a_{1}^{2}-32 r_{0} a_{1}^{2}+24 r_{0}^{2}+36}{24 r_{0}\left(2 r_{0}^{2}+1\right)}, \\
& c_{2}=\frac{6 r_{0}^{4} a_{1}^{4}+105 r_{0}^{3} a_{1}^{2}+32 r_{0} a_{1}^{2}-24 r_{0}^{2}-36}{4 r_{0}^{2}\left(2 r_{0}^{2} a_{1}^{2}+3\right)} .
\end{aligned}
$$

In our actual numerical calculations, we have expanded up to and including the $\left(r-r_{0}\right)^{4}$ order. These expansions are then used in order to set initial conditions just outside the horizon. We then integrate out to large $r$. The criterion for a good black hole solution is that the metric and Proca functions should approach the asymptotic forms given in (1.7). We find that indeed such solutions arise, and they are stable as the parameters $r_{0}$ and $a_{1}$ are adjusted.

As we did in the case of the solitonic solutions, here too we can attempt a numerical confirmation that these black hole solutions obey the first law of thermodynamics that we derived in section 4 . Taking the simple choice $\gamma=0$ again, the first law is given by (4.14). We shall present an example calculation in $n=5$ dimensions, for which the first law becomes

$$
d M=T d S-\frac{1}{2} \pi q_{1} d q_{2} .
$$



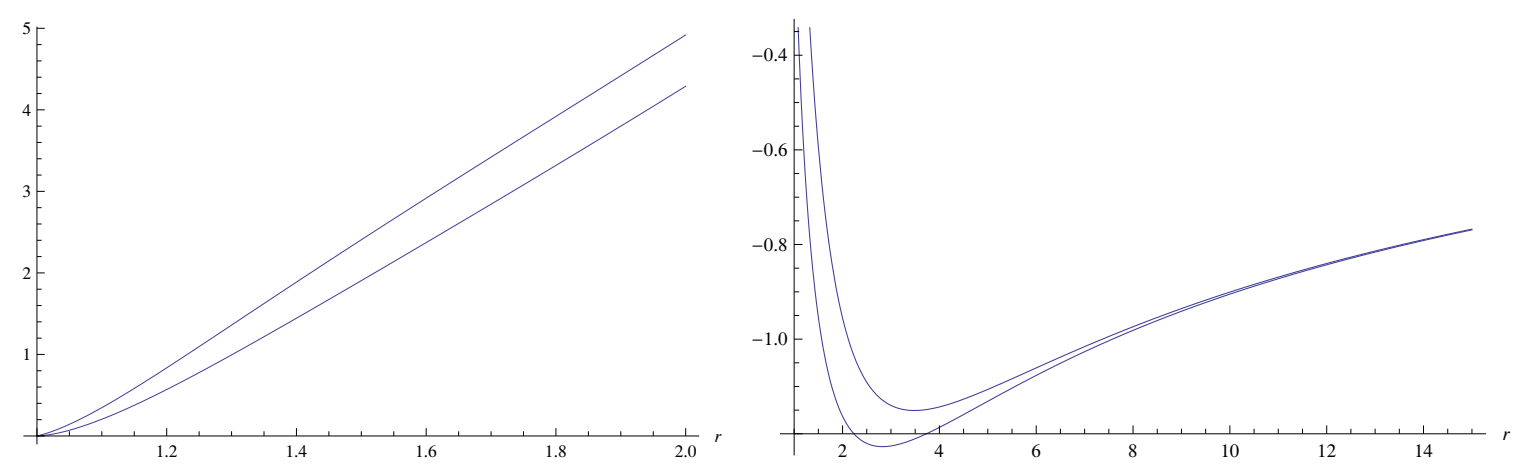

Figure 3. Black hole in $n=5$ dimensions, with $a_{1}=-10$ and $b_{1}=2.163$. The left-hand plot shows the metric functions $h(r)$ and $f(r)$. The upper line is $h(r)$, starting from $h\left(r_{0}\right)=0$, and the lower line is $f(r)$, starting from $f\left(r_{0}\right)=0$. To leading order, they coalesce at large $r$, which we did not present in this figure. The right-hand plot shows the potential function $\psi$ (lower line), and compares it with a best fit of the function $\tilde{\psi}=q_{1} / r^{\frac{1}{2}}+q_{2} / r^{\frac{3}{2}}$ (upper line) that represents the leading-order terms in the large- $r$ expansion in (6.2), achieved by taking $q_{1}=-3.222$ and $q_{2}=3.742$. The actual numerically-obtained potential function $\psi$ vanishes at small $r=r_{0}$, although $\tilde{\psi}\left(r_{0}\right) \neq 0$.

The easiest case to consider is when we fix the entropy. This corresponds to holding $r_{0}$ fixed, and the first law becomes

$$
d M=T d S-\frac{1}{2} \pi q_{1} d q_{2} \quad \longrightarrow \quad d M=-\frac{1}{2} \pi q_{1} d q_{2}
$$

As a concrete example, let us set $r_{0}=1$. The solution then has one non-trivial adjustable parameter, $a_{1}$, remaining. The parameter $b_{1}$ should be fixed so that we have $\left.\frac{h}{r^{2}}\right|_{r \rightarrow \infty}=1$ at large $r$. To see in more detail how the metric function and $\psi$ behave, let us take as an example $a_{1}=-10$, implying $c_{1}=18 / 203$. We find that we should then take $b_{1}=2.163$. Thus the black hole has a temperature

$$
T=\frac{b_{1} c_{1}}{4 \pi}=0.01526 \text {. }
$$

The plots for the metric functions $(h, f)$ and the Proca potential $\psi$ are given in figure 3 . From the numerical solution, we can read off $m_{2}=-23.93$ and $\left(q_{1}, q_{2}\right)=(-3.222,3.742)$, and hence find that the mass is given by $M=11.63$.

We can now solve numerically for a range of values for the parameter $a_{1}$ and hence read off $\left(q_{1}, q_{2}, M\right)$ and the temperature, all depending on the chosen values of $a_{1}$. In particular, when $a_{1}=0$, the solution becomes the usual Schwarzschild AdS-black hole, with $r_{0}=1$. Here we present the results for $a_{1}$ in the range $-\frac{3}{4} \leq a_{1} \leq 0$. We can then express the quantities $\left(M, q_{1}, T\right)$ as functions of $q_{2}$. The results are given in figures 4 and 5 .

The data fitting for small $q_{2}$ implies that

$$
M=\frac{1}{2} \pi\left(\frac{3}{2}+0.420 q_{2}^{2}\right), \quad q_{1}=-0.843 q_{1} .
$$

Note that

$$
-\frac{2}{\pi} \frac{\partial M}{\partial q_{2}}=-0.840 q_{2}
$$



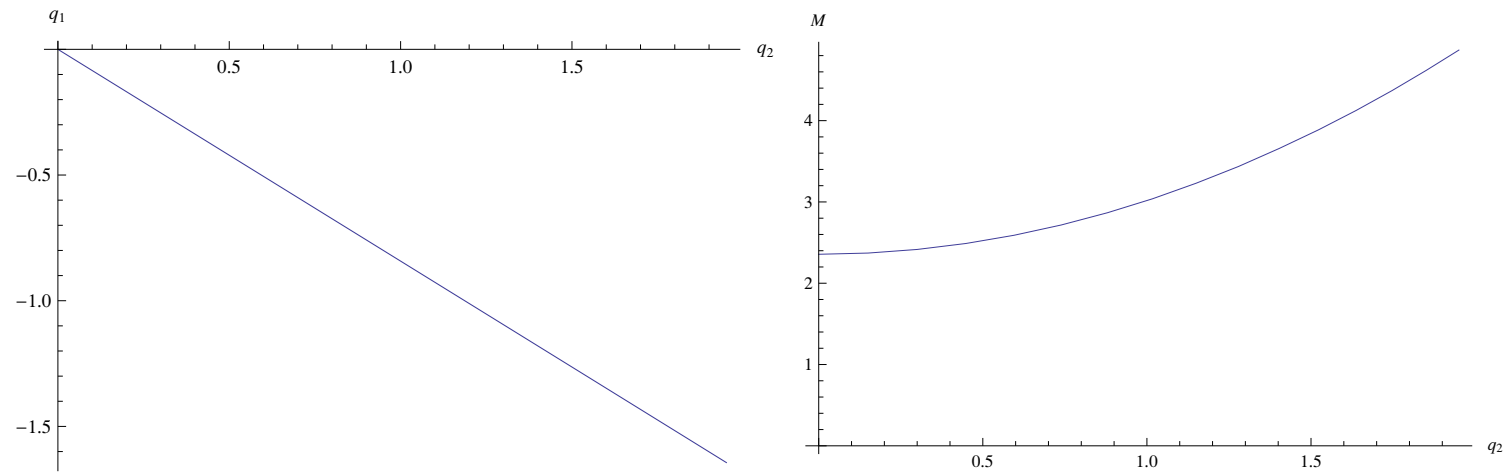

Figure 4. Black hole for $r_{0}=1$ and $0<q_{2}<2$. The solution is Schwarzschild-AdS when $q_{2}=0$. For small $q_{2}$, the function $q_{1}\left(q_{2}\right)$ is linear and $M\left(q_{2}\right)$ is parabolic.

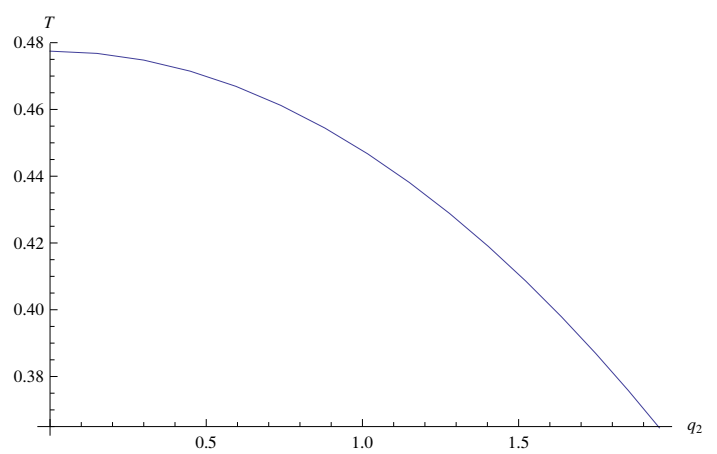

Figure 5. Temperature of the black hole for $r_{0}=1$ and $0<q_{2}<2$.
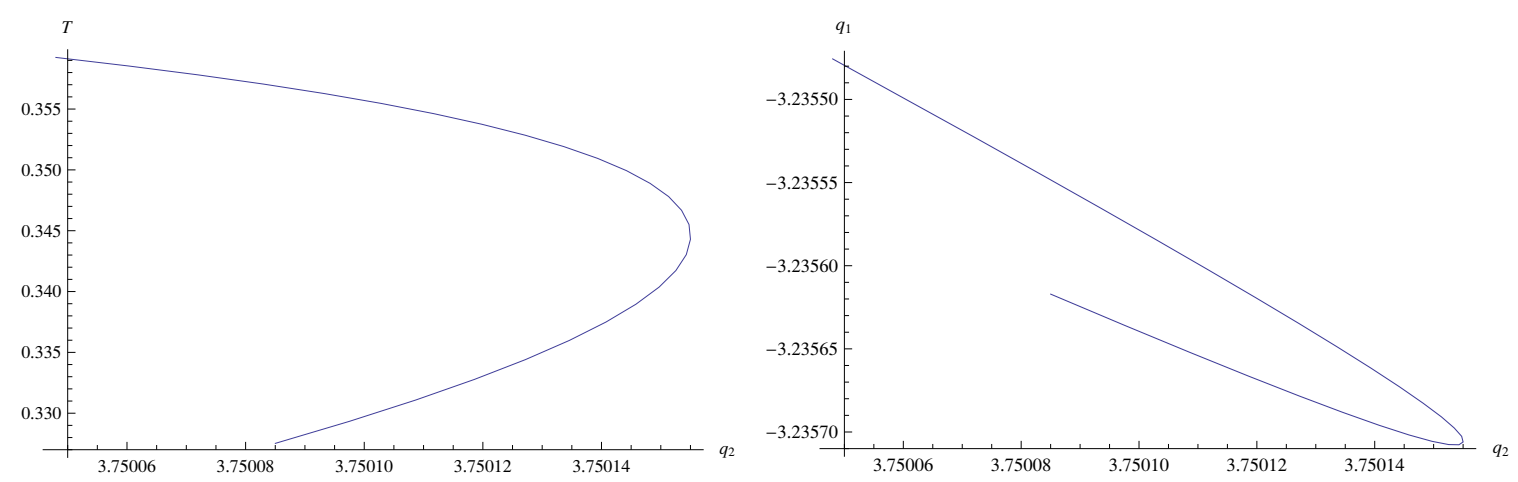

Figure 6. Black hole for $r_{0}=1$ and larger $q_{2}$. Note that for a given $q_{2}$, there can be two values for $q_{1}$ or $T$ respectively, suggesting that a phase transition can occur.

If we decrease $a_{1}$ from 0 to negative values, eventually the solution becomes singular when $a_{1}$ reaches about $a_{1}^{*} \sim-20.12$. We obtained data from $a_{0}=-18$ to $a_{1}=-20$, and the results for $T$ and $q_{1}$ are plotted in figure 6 .

What is curious is that as $a_{1}$ approaches $a_{1}^{*}$, the parameters $\left(q_{1}, q_{2}, m_{2}\right)$ remain finite, approaching certain fixed values. Yet the solution becomes singular once $a_{1}$ passes over $a_{1}^{*}$. 


\section{Conclusions}

In this paper, we have investigated the static spherically-symmetric solutions of the theory of a massive Proca field coupled to gravity, in the presence of a negative cosmological constant. The fact that the solutions are asymptotic to anti-de Sitter, rather than Minkowski, spacetime has a profound effect on their geometry and stability. In the absence of a cosmological constant, a generic static spherically-symmetric solution of the Proca equation will take the form $\psi=A_{0} \sim \alpha e^{-\tilde{m} r} / r^{n-3}+\beta e^{\tilde{m} r} / r^{n-3}$, and so without a fine-tuning to set $\beta=0$, the solution will diverge exponentially at infinity. There will in turn be backreaction on the metric that leads to analogous singular behaviour. This implies that even if one finds a solution that is well behaved on the horizon of a black hole, its evolution out to large $r$ will inevitably pick up some component of the diverging asymptotic solution, thus implying that it will be singular. The asymptotic solutions would instead be decaying and oscillatory in $r$ if $\tilde{m}^{2}$ were negative, but then, the Proca field would be tachyonic and so the solutions would exhibit runaway behaviour with real exponential time dependence.

By contrast, with the cosmological constant turned on, two factors come into play that radically change the picture. First of all, the asymptotic behaviour of the Proca solutions in the AdS background involve power-law rather than exponential dependence on $r$. Secondly, there is now a window of negative mass-squared values for the Proca mass, extending in the range $\mathbf{m}_{\mathrm{BF}}^{2} \leq \tilde{m}^{2}<0$, where $\mathbf{m}_{\mathrm{BF}}^{2}$ is the Breitenlohner-Freedman bound given in (1.4), within which the Proca field is still non-tachyonic, and thus is not subject to exponential time-dependent runaway behaviour. Between them, these two factors imply that perfectly well-behaved black hole solutions exist, provided that the Proca mass-squared lies in an appropriate range. There also exist solitonic solutions, that extend smoothly to an asymptotically AdS region from an origin of the radial coordinate at $r=0$.

We performed some numerical integrations to demonstrate the existence of such wellbehaved black hole and solitonic solutions, but in fact, one can see on general grounds that they must exist. Namely, by making a general expansion of the Proca and metric functions in the vicinity of the horizon, in the black hole case, or of the origin, in the solitonic case, one can first establish that well-behaved short-distance solutions exist in each case. Although one does not know precisely how these join on to the solutions at large distance, which are known only asymptotically, the fact that the general asymptotic solutions are well-behaved means that the evolution from small to large $r$ will necessarily be a smooth one. In other words, there is no issue in this asymptotically-AdS situation of needing a "fine-tuning" to avoid an evolution to a singular solution at infinity, since all large- $r$ solutions are non-singular.

The calculation of the mass of the static spherically-symmetric solutions can be somewhat delicate, because the way in which they approach AdS at infinity may involve fall-offs that are slower than in a typical AdS black hole such as Schwarzschild-AdS. In the case of Schwarzschild-AdS, the metric functions $h$ and $f$ in (2.4) take the form $h=f=r^{2} \ell^{-2}+1-m / r^{n-3}$ in $n$ dimensions, and one might think that any approach to pure AdS that was slower than the $1 / r^{n-3}$ rate would lead to a diverging "mass". We performed our calculations of the mass using the renormalised holographic stress tensor, 
and it turns out that when the contribution from the Proca field is properly taken into account, the result in general is perfectly finite and well defined. We also carried out a derivation of the first law of thermodynamics using the techniques developed by Wald, and we found that this gives consistent and meaningful results.

An important feature in the first law is that there is a contribution from the Proca field, giving a result typically of the form $d M=T d S+$ (const) $q_{1} d q_{2}$, where $q_{1}$ and $q_{2}$ are the two arbitrary coefficients in the asymptotic form of the Proca solution. One might think of $q_{2}$ as being like a "charge" for the Proca field and $q_{1}$ as a conjugate "potential". However, since $q_{2}$ is not associated with a conserved quantity it is not necessarily clear whether it should really be thought of as a "charge", or whether instead it should be viewed as being a parameter characterising a Proca "hair". In any case, the inclusion of the $q_{1} d q_{2}$ term is necessary in order to obtain an integrable first law. The situation is in fact analogous to one that was encountered for the gauged dyonic black hole in [12], where a careful examination of the contribution of a scalar field in the first law showed that it gave an added contribution, with the leading coefficients in the asymptotic expansion of the scalar field being the extra thermodynamic variables.

\section{Acknowledgments}

We are grateful to Sijie Gao and Yi Pang for useful discussions. H-S.L. is supported in part by NSFC grant 11305140 and SFZJED grant Y201329687. The research of H.L. is supported in part by NSFC grants 11175269 and 11235003 . The work of C.N.P. is supported in part by DOE grant DE-FG02-13ER42020.

\section{A Proca solutions in $\operatorname{AdS}_{n}$, and the Breitenlohner-Freedman bound}

Here we record some results for spherically-symmetric solutions of the Proca equation in a pure $\mathrm{AdS}_{n}$ background metric. These allow us to study the asymptotic form of the Proca field in our black hole and soliton solutions, and also to give a simple derivation of the Breitenlohner-Freedman bound for massive vector modes in $n$-dimensional anti-de Sitter spacetime.

From the last equation in (2.6), we see that in a pure AdS background, which has $h=f=1+r^{2} \ell^{-2}$, the Proca potential $\psi$ for a static spherically-symmetric field $A=\psi(r) d t$ satisfies the equation

$$
\frac{1}{r^{n-2}}\left(1+r^{2} \ell^{-2}\right)\left(r^{n-2} \psi^{\prime}\right)^{\prime}=\tilde{m}^{2} \psi .
$$

This can be solved straightforwardly in terms of hypergeometric functions, giving

$$
\begin{aligned}
\psi= & \frac{q_{1}}{r^{(n-3-\sigma) / 2}} F\left(\frac{3-n-\sigma}{4}, \frac{n-3-\sigma}{4}, \frac{2-\sigma}{2},-\frac{\ell^{2}}{r^{2}}\right) \\
& +\frac{q_{2}}{r^{(n-3+\sigma) / 2}} F\left(\frac{3-n+\sigma}{4}, \frac{n-3+\sigma}{4}, \frac{2+\sigma}{2},-\frac{\ell^{2}}{r^{2}}\right),
\end{aligned}
$$

where

$$
\sigma=\sqrt{4 \tilde{m}^{2} \ell^{2}+(n-3)^{2}}
$$


and $\tilde{m}$ is the mass of the Proca field. (Since our focus is principally on the large- $r$ asymptotic behaviour of the solutions, we have presented them in the form where the hypergeometric functions are analytic functions of $1 / r^{2}$.) Note that the leading-order terms $q_{1} r^{(3+\sigma-n) / 2}$ and $q_{2} r^{(3-\sigma-n) / 2}$ associated with the two independent solutions then have "descendants" falling off with the additional factors of integer powers of $1 / r^{2}$.

The Breitenlohner-Freedman bound for the vector modes is determined by the requirement that the parameter $\sigma$ appearing in (A.2) should be real. Thus from (A.3) we see that the bound is given by

$$
\tilde{m}^{2} \geq m_{\mathrm{BF}}^{2} \equiv-\frac{(n-3)^{2}}{4 \ell^{2}} .
$$

A case of particular interest in this paper is when the Proca mass $\tilde{m}$ is chosen to be given by (2.7), in order to ensure that the sequence of terms in the power-series expansion of $\psi(r)$ at large $r$ should involve inverse powers of $r$ that increase in steps of $1 / r$. Thus we see from (A.4) that our mass parameter $\tilde{m}$ lies within the Breitenlohner-Freedman bound, with

$$
\tilde{m}^{2}=m_{\mathrm{BF}}^{2}+\frac{1}{4 \ell^{2}}>m_{\mathrm{BF}}^{2} .
$$

We then study a more extended range of values for $\tilde{m}$.

Note that in the limit where the cosmological constant goes to zero (i.e. $\ell \rightarrow \infty$ ), the Proca solution (A.2) becomes

$$
\psi=\frac{\alpha e^{-\tilde{m} r}}{r}+\frac{\beta e^{\tilde{m} r}}{r} .
$$

Unlike the AdS case, where both solutions of the Proca equation can be well-behaved at infinity, in an asymptotically-Minkowski background one of the solutions always diverges exponentially.

Open Access. This article is distributed under the terms of the Creative Commons Attribution License (CC-BY 4.0), which permits any use, distribution and reproduction in any medium, provided the original author(s) and source are credited.

\section{References}

[1] J.M. Maldacena, The large- $N$ limit of superconformal field theories and supergravity, Adv. Theor. Math. Phys. 2 (1998) 231 [Int. J. Theor. Phys. 38 (1999) 1113] [hep-th/9711200] [INSPIRE].

[2] S.S. Gubser, I.R. Klebanov and A.M. Polyakov, Gauge theory correlators from noncritical string theory, Phys. Lett. B 428 (1998) 105 [hep-th/9802109] [INSPIRE].

[3] E. Witten, Anti-de Sitter space and holography, Adv. Theor. Math. Phys. 2 (1998) 253 [hep-th/9802150] [INSPIRE].

[4] P. Breitenlohner and D.Z. Freedman, Positive energy in anti-de Sitter backgrounds and gauged extended supergravity, Phys. Lett. B 115 (1982) 197 [INSPIRE].

[5] P. Breitenlohner and D.Z. Freedman, Stability in gauged extended supergravity, Annals Phys. 144 (1982) 249 [INSPIRE]. 
[6] H. Lü, Y. Pang, C.N. Pope and J.F. Vazquez-Poritz, AdS and Lifshitz black holes in conformal and Einstein-Weyl gravities, Phys. Rev. D 86 (2012) 044011 [arXiv:1204.1062] [INSPIRE].

[7] S. Kachru, X. Liu and M. Mulligan, Gravity duals of Lifshitz-like fixed points, Phys. Rev. D 78 (2008) 106005 [arXiv:0808.1725] [InSPIRE].

[8] P. Kovtun and D. Nickel, Black holes and non-relativistic quantum systems, Phys. Rev. Lett. 102 (2009) 011602 [arXiv:0809.2020] [InSPIRE].

[9] M. Taylor, Non-relativistic holography, arXiv:0812.0530 [INSPIRE].

[10] A. Ashtekar and A. Magnon, Asymptotically anti-de Sitter space-times, Class. Quant. Grav. 1 (1984) L39 [InSPIRE].

[11] A. Ashtekar and S. Das, Asymptotically anti-de Sitter space-times: conserved quantities, Class. Quant. Grav. 17 (2000) L17 [hep-th/9911230] [INSPIRE].

[12] H. Lü, Y. Pang and C.N. Pope, AdS dyonic black hole and its thermodynamics, JHEP 11 (2013) 033 [arXiv: 1307.6243] [INSPIRE].

[13] H.-S. Liu and H. Lü, Scalar charges in asymptotic AdS geometries, Phys. Lett. B 730 (2014) 267 [arXiv:1401.0010] [INSPIRE].

[14] V. Balasubramanian and P. Kraus, A stress tensor for anti-de Sitter gravity, Commun. Math. Phys. 208 (1999) 413 [hep-th/9902121] [INSPIRE].

[15] R.C. Myers, Stress tensors and Casimir energies in the AdS/CFT correspondence, Phys. Rev. D 60 (1999) 046002 [hep-th/9903203] [INSPIRE].

[16] R. Emparan, C.V. Johnson and R.C. Myers, Surface terms as counterterms in the AdS/CFT correspondence, Phys. Rev. D 60 (1999) 104001 [hep-th/9903238] [INSPIRE].

[17] S. de Haro, S.N. Solodukhin and K. Skenderis, Holographic reconstruction of space-time and renormalization in the AdS/CFT correspondence, Commun. Math. Phys. 217 (2001) 595 [hep-th/0002230] [INSPIRE].

[18] P. Kraus, F. Larsen and R. Siebelink, The gravitational action in asymptotically AdS and flat space-times, Nucl. Phys. B 563 (1999) 259 [hep-th/9906127] [INSPIRE].

[19] R.M. Wald, Black hole entropy is the Noether charge, Phys. Rev. D 48 (1993) 3427 [gr-qc/9307038] [INSPIRE].

[20] V. Iyer and R.M. Wald, Some properties of Noether charge and a proposal for dynamical black hole entropy, Phys. Rev. D 50 (1994) 846 [gr-qc/9403028] [INSPIRE].

[21] S. Gao, The first law of black hole mechanics in Einstein-Maxwell and Einstein-Yang-Mills theories, Phys. Rev. D 68 (2003) 044016 [gr-qc/0304094] [rNSPIRE]. 